This document is the Accepted Manuscript version of a Published Work that appeared in final form in a Nature Publishing Group (NPG) Journal after peer review and technical editing by the publisher.

\title{
Synuclein strains cause distinct synucleinopathies after local and systemic administration
}

\author{
Authors: W. Peelaerts ${ }^{1}$, L. Bousset ${ }^{2}$, A. Van der Perren'1, A. Moskalyuk ${ }^{3}$, R. Pulizzi ${ }^{3}$, \\ M. Giugliano ${ }^{3,4,5}$, C. Van den Haute ${ }^{1,6}$, R. Melki $^{2}$, V. Baekelandt ${ }^{1}$
}

\begin{abstract}
Affiliations:
${ }^{1} \mathrm{KU}$ Leuven, Laboratory for Neurobiology and Gene Therapy, Department of Neurosciences, 3000 Leuven, Belgium.

2 Paris-Saclay Institute of Neuroscience, CNRS, Avenue de la Terrasse, 91198 Gif-surYvette, France.

3 Theoretical Neurobiology \& Neuroengineering Laboratory, Department of Biomedical Sciences, University of Antwerp, Belgium;

${ }^{4}$ Department of Computer Science, University of Sheffield, United Kingdom

${ }^{5}$ Brain Mind Institute, Swiss Federal Institute of Technology of Lausanne, Switzerland.

${ }^{6}$ KU Leuven, Leuven Viral Vector Core, 3000 Leuven, Belgium.
\end{abstract}

Nature. 2015 Jun 10.

doi: $10.1038 /$ nature14547

To access the final edited and published article see

http://www.nature.com/nature/journal/vaop/ncurrent/full/nature14547.html 


\title{
Synuclein strains cause distinct synucleinopathies after local and systemic administration
}

\author{
Authors: W. Peelaerts ${ }^{1}$, L. Bousset ${ }^{2}$, A. Van der Perren ${ }^{1}$, A. Moskalyuk ${ }^{3}$, R. Pulizzi ${ }^{3}$, M. \\ Giugliano $^{3,4,5}$ C. Van den Haute ${ }^{1,6}$, R. Melki ${ }^{2 *}$, V. Baekelandt ${ }^{1}$ *
}

\author{
Affiliations: \\ ${ }^{1}$ KU Leuven, Laboratory for Neurobiology and Gene Therapy, Department of \\ Neurosciences, 3000 Leuven, Belgium. \\ ${ }^{2}$ Paris-Saclay Institute of Neuroscience, CNRS, Avenue de la Terrasse, 91198 Gif-sur- \\ Yvette, France. \\ ${ }^{3}$ Theoretical Neurobiology \& Neuroengineering Laboratory, Department of Biomedical \\ Sciences, University of Antwerp, Belgium; \\ ${ }^{4}$ Department of Computer Science, University of Sheffield, United Kingdom \\ ${ }^{5}$ Brain Mind Institute, Swiss Federal Institute of Technology of Lausanne, Switzerland. \\ ${ }^{6}$ KU Leuven, Leuven Viral Vector Core, 3000 Leuven, Belgium.
}

\section{Summary Paragraph}

Misfolded protein aggregates represent a continuum with overlapping features in neurodegenerative diseases but differences in protein components and affected brain regions ${ }^{1}$. The molecular hallmark of synucleinopathies such as Parkinson's Disease (PD), Dementia with Lewy Bodies (DLB) and Multiple System Atrophy (MSA) are megadalton $\alpha$-synuclein-rich deposits suggestive of one molecular event causing distinct disease phenotypes. Glial $\alpha$-synuclein $(\alpha \mathrm{SYN})$ filamentous deposits are prominent in MSA while neuronal $\alpha S Y N$ inclusions are found in PD and DLB ${ }^{2}$. The discovery of $\alpha S Y N$ assemblies with different structural characteristics or 'strains' has led to the hypothesis that strains could account for the different clinico-pathological traits within synucleinopathies ${ }^{3,4}$. In this study we show that $\alpha$ SYN strain conformation and seeding propensity lead to distinct histopathological and behavioral phenotypes. We assess the properties of structurally well-defined $\alpha \mathrm{SYN}$ assemblies (oligomers, ribbons and fibrils) after injection in rat brain. We prove that $\alpha \mathrm{SYN}$ strains amplify in vivo. Fibrils appear as 
the major toxic species resulting in progressive motor impairment and cell death, while ribbons cause a distinct histopathological phenotype displaying PD and MSA traits. Additionally, we show that $\alpha \mathrm{SYN}$ assemblies cross the blood-brain barrier and distribute to the central nervous system after intravenous injection. Our results demonstrate that distinct aSYN strains display differential seeding capacities inducing strain-specific pathology and neurotoxic phenotypes.

\section{Main Text}

The discovery that LB spread within the nervous system ${ }^{5,6}$ led to the demonstration that fibrillar assemblies seed soluble $\alpha \mathrm{SYN}$ and spread between cells in cellular and animal models ${ }^{7}$. The most widely accepted paradigm postulates that prefibrillar oligomers, as opposed to mature fibrils, represent the neurotoxic entities in $\mathrm{PD}^{8,9}$. Although a direct quantitative in vivo comparison of the neurotoxic potential of different $\alpha \mathrm{SYN}$ assemblies is lacking, recent evidence indicates that fibrillar $\alpha \mathrm{SYN}$ toxicity is significantly greater than that of pre-fibrillar precursors ${ }^{4,10}$. Studies with inoculation of recombinant fibrils or pathogenic brain lysates in rodent brain triggered $\alpha \mathrm{SYN}$ pathology with varying efficiencies and moderate neuronal loss ${ }^{11,12}$. In contrast, recombinant adenoassociated viral vector-mediated (rAAV) overexpression of $\alpha \mathrm{SYN}$ in rodent and primate brain induces time-dependent robust behavioral impairment and dopaminergic (DA) neurodegeneration $^{13,14}$. In this study, we compared the in vivo properties of $\alpha \mathrm{SYN}$ oligomers and two distinct $\alpha$ SYN strains ('fibrils' and 'ribbons') (Extended Data Fig. 1ae $)^{4}$, by inoculation of highly purified and structurally characterized recombinant human $\alpha \mathrm{SYN}$ preparations in rat substantia nigra (SNpc) in absence and presence of rAAVmediated human $\alpha \mathrm{SYN}$ overexpression.

To assess $\alpha \mathrm{SYN}$ uptake and spreading in a detailed and quantitative way, we fluorescently labeled all $\alpha$ SYN assemblies (Extended Data Fig. 1f-g). First, we applied ex vivo fluorescent tomography to visualize spreading of $\alpha \mathrm{SYN}$ in whole brain after inoculating $10 \mu \mathrm{g}$ of $\alpha \mathrm{SYN}$ assemblies (Fig. 1a and Extended data Table 1). Reconstructed images of the fluorescent volume (FV) after 20 minutes and 7 days showed that $\alpha \mathrm{SYN}$ oligomers spread more efficiently than fibrils, ribbons and free fluorophore (Fig. 1b). Subsequent histological analysis revealed that all assemblies were taken up in DA neurons, spread over the arborizing striatal DA axons and were 
transmitted trans-synaptically in a time-dependent manner (Extended Data Fig. 2a-g). These results build upon previous findings that the $\alpha \mathrm{SYN}$ oligomers disseminate more efficiently than higher molecular weight assemblies after intracerebral inoculation ${ }^{15,16}$.

The reason why certain LB-bearing regions are subject to neurodegeneration and others not, remains incompletely understood. The fact that PD, DLB and MSA patients present variable striatonigral pathology is also confounding. We therefore assessed whether $\alpha$ SYN oligomers, fibrils and ribbons translate their distinctive structural and biochemical properties into motor behavioral and neurotoxic phenotypes. Four months after nigral injection of ribbons or fibrils LB- and LN-like inclusions formed essentially in DA neurons. These juxta-, peri-, nuclear and filamentous inclusions stained positive for $\alpha$ SYN phosphorylated at Ser129 (P $\alpha \mathrm{SYN})$, resembling in that to $\mathrm{LB} / \mathrm{LN}$ pathologyassociated inclusions (Fig. 1c-f and Extended Data Fig. 3). P $\alpha$ SYN inclusions were considerably more abundant for $\alpha \mathrm{SYN}$ ribbons compared to fibrils with a predominant LN-like filamentous phenotype staining positive for the aggresome marker p62/SQSTM1 (Fig. 1e-g). Injection of $\alpha \mathrm{SYN}$ oligomers and brain homogenate from aged 18-month transgenic mouse expressing human A30P $\alpha \mathrm{SYN}$ did not result in detectable P $\alpha \mathrm{SYN}$ accumulation. Combined rAAV-mediated $\alpha \mathrm{SYN}$ overexpression with ribbons and fibrils inoculation lead to increased $\mathrm{P} \alpha \mathrm{SYN}^{+}$cells (Extended Data Fig. 4). Strikingly, and only after combined rAAV-mediated $\alpha S Y N$ overexpression with $\alpha S Y N$ ribbons inoculation, a second but sparse $\alpha \mathrm{SYN}$ phosphorylation pattern was observed in oligodendroglial cells (Fig 1h-i).

In terms of neurotoxicity, nigral inoculation of brain homogenate, oligomers, fibrils and ribbons did not induce detectable DA cell death until the final time point of 120 days (fig. 2a-b). In contrast, sole rAAV-mediated $\alpha \mathrm{SYN}$ overexpression (control) led to $27 \%$ cell loss of $\mathrm{TH}^{+}$neurons and $32 \%$ striatal DA axonal loss (fig. 2c, d). Combined rAAV- $\alpha S Y N$ overexpression with inoculation of brain homogenate, $\alpha \mathrm{SYN}$ oligomers, fibrils or ribbons accelerated $\mathrm{TH}^{+}$cell death (fig. 2c-e) and severely reduced striatal DA nerve terminal volume by 61 and $43 \%$ for $\alpha \mathrm{SYN}$ fibrils and brain homogenate, respectively. Combined rAAV- $\alpha \mathrm{SYN}$ overexpression and inoculation of $\alpha \mathrm{SYN}$ oligomers and ribbons did not yield additional TH fiber loss (fig. 2d). Assessment of spontaneous forepaw use with the cylinder test revealed a consistent significant motor deficit from 60 
days onwards upon injection of $\alpha \mathrm{SYN}$ fibrils (fig. 2f). Combined rAAV-mediated $\alpha \mathrm{SYN}$ overexpression with $\alpha \mathrm{SYN}$ assemblies inoculation further aggravated motor deficits (Fig. $2 \mathrm{~g}$ ). These findings demonstrate that introduction of seeds under conditions where $\alpha \mathrm{SYN}$ is expressed at higher levels greatly enhances neurodegeneration in a strain-dependent manner. Thus, although ribbon inoculation induced more pronounced LB/LN-like inclusions (Fig. 1c-f), fibrils imposed the largest neurotoxic burden on the striatonigral pathway (Fig. 2h).

The observation of a behavioral phenotype after inoculation of pure $\alpha \mathrm{SYN}$ strains in the absence of DA degeneration indicated that this functional deficit was not a downstream effect of DA cell death. Recent studies in cellular models, $\alpha \mathrm{SYN}$ transgenic animals and patients with incidental LB disease, PD and DLB have shown neuronal dysfunction and motor abnormalities before overt neurodegeneration and LB/LN pathology ${ }^{17-19}$. We therefore employed cellular electrophysiology to study the impact of $\alpha S Y N$ assemblies on neuronal and synaptic physiology. Endogenous $\alpha / \beta S Y N$ is calculated to be over $40 \mu \mathrm{M}$ within the rat synaptosome ${ }^{20}$. Four days exposure to $1 \mu \mathrm{M}$ of $\alpha \mathrm{SYN}$ assemblies to rat primary cortical cultures left passive and excitable electrical membrane properties unaltered in single neurons, as recorded intracellularly by patchclamp (Extended Data Fig. 5a-e). Instead, significant effects were observed on synaptic physiology. The frequency of occurrence of spontaneous bursts of action potentials (APs) revealed a direct substantial decrease after exposure to aSYN assemblies (Fig. 2i-j). Further evidence was obtained via substrate-integrated microelectrode arrays (MEAs) allowing simultaneous extracellular monitoring of the spontaneous neuronal network electrical activity from 59 independent microelectrodes. The spontaneous firing of cortical neurons self-organize ex vivo as episodic network-wide epochs of AP synchrony, offering an indirect indicator of (dys)functional glutamatergic synaptic transmission. Exposure to low concentrations of different $\alpha \mathrm{SYN}$ assemblies revealed an increase in the average inter-spike intervals, which was significant for fibrils and oligomers (Figure 2k$1)$.

Taken together, these data indicate that different $\alpha \mathrm{SYN}$ assemblies can affect neurotransmission after acute exposure, but only fibrillar $\alpha \mathrm{SYN}$ exhibited perpetual behavioral and aggravated neurotoxic phenotypes in vivo. To further investigate this, we 
assessed the capacities of $\alpha \mathrm{SYN}$ oligomers, ribbons and fibrils to seed endogenous $\alpha \mathrm{SYN}$ and to propagate, leading to long-term functional effects. We inoculated $40 \mu \mathrm{g}$ of $\alpha \mathrm{SYN}$ assemblies divided over 3 injection sites in rat striatum in combination with rAAV- $\alpha \mathrm{SYN}$ overexpression (Fig. 3a) or not (Extended Fig. 6). Large amounts of sarkosyl-insoluble rodent $\alpha$ SYN were detected after 60 days throughout the striatonigral pathway for ribbons and fibrils (Fig. 3a and Extended Fig. 6) although the inoculated human $\alpha \mathrm{SYN}$ was undetected after sarkosyl extraction in animals injected only with $\alpha \mathrm{SYN}$ assemblies (Extended Data Fig. 7). This implies that exogenous $\alpha \mathrm{SYN}$ strains seed the assembly of endogenous $\alpha \mathrm{SYN}$ overcoming the species barrier. In addition, the Proteinase $\mathrm{K}$ resistance of sarkosyl-insoluble $\alpha$ SYN extracted from the brains of animals overexpressing $\alpha \mathrm{SYN}$ inoculated with either ribbons or fibrils differed (Fig 3b). Fibrils exhibited a higher resistance compared to ribbons reflecting the capacity of each strain to amplify in a specific manner in vivo (Fig. 3b and Extended Data Fig. 6 and 7). Hence, fibrils possess the highest capacity to translate their neuropathological potential from a defined structural to a wide spatio-temporal level.

$\alpha S Y N$ knockout animals show no robust pathology possibly because of functional redundancy $(\beta \text { - and } \mathrm{y} \text {-SYN isoforms })^{21}$. The remarkable capacity of $\alpha \mathrm{SYN}$ strains to persist and act as seeds by imprinting their intrinsic structures causing synaptic defects argues for a gain-of-toxic function. $\alpha \mathrm{SYN}$ fibrils can multiply by simple elongation with addition of monomers at their ends or by breakage creating additional nuclei ${ }^{22,23}$. This growth process appears strain-dependent, making strains the crucial pathogenic factor. Indeed, several months after nigral injection and in sharp contrast to all other assemblies, $\alpha S Y N$ fibrils were detectable in striatal DA axons (Extended Data Fig. 8). Therefore, and although all $\alpha \mathrm{SYN}$ species might play a role during pathogenesis, fibrillar $\alpha \mathrm{SYN}$ exhibited the largest neurotoxic potential accompanied by disease-specific hallmarks at long-term (overview in Extended data Table 2).

In early stages of neurodegeneration pathological changes are often localized in defined brain areas while at later stages neuronal dysfunction and associated protein aggregates are widespread. Oligomeric prion proteins invade the CNS acutely after crossing the blood brain-barrier $(\mathrm{BBB})$ or chronically via the splenic-nerve route ${ }^{24}$. To assess whether $\alpha \mathrm{SYN}$ assemblies cross the $\mathrm{BBB}$, or behave in a similar manner, we 
repeatedly injected $\alpha \mathrm{SYN}$ oligomers, ribbons and fibrils intravenously on a two-weekly basis during 4 months using 2 fluorescent labels, providing additional temporal resolution (Fig. 4a). No rAAV- $\alpha$ SYN was used in these experiments. The red-shifted fluorescent label allowed epifluorescence imaging of the injected $\alpha \mathrm{SYN}$ species in live animals. At the final time point we observed a striking, localized fluorescent signal originating from the brain for $\alpha \mathrm{SYN}$ oligomers, ribbons and fibrils but not for control animals injected with saline or the free atto-647 label (Fig. 4b-c). Immunohistochemical staining revealed aSYN deposits in the CNS for ribbons and fibrils (Fig. 4d-e and Extended Data Fig. 9) but only diffuse staining for oligomers (Fig 4d, Extended Data Fig. 10 a-f). This could indicate that $\alpha \mathrm{SYN}$ oligomers might either diffuse to a higher extent because of their molecular mass or are partly washed out during perfusion, while $\alpha \mathrm{SYN}$ fibrils and ribbons remain in place after crossing the BBB. Detailed histological analysis showed an accumulation of atto-550 and atto-647 labeled human $\alpha \mathrm{SYN}$ fibrils in cortical neurons (Fig. 4f) and in spinal cord (Fig. 4g). This pattern was accompanied with microglial activation, which was most pronounced for fibrils throughout the spinal cord, and with redistribution of the early neuronal injury marker GAP43 (Fig. 4d-e and Extended Data Fig. 10g). These observations demonstrate that in this experimental setup the spread of $\alpha \mathrm{SYN}$ assemblies is not restricted to the intracerebral route but extends beyond the CNS.

The findings that $\alpha \mathrm{SYN}$ spread after intramuscular and gastric injection ${ }^{25,26}$, and the demonstration of systemic spread we make here are striking. The contribution of these routes to $\alpha \mathrm{SYN}$ assemblies spread and associated pathology deserves further investigation. In contrast to rapidly transmitted oligomers, the in vivo behavior of slowly amplifying aSYN strains may provide a basis for the observed heterogeneity in synucleinopathies and its slow but relentless pattern of spreading. This underlines the need for therapeutic strategies aimed at changing the surface properties of $\alpha \mathrm{SYN}$ assemblies either using epitope- or strain-specific antibodies or molecular chaperonederived peptides ${ }^{27}$ to interfere with the propagation of disease-associated $\alpha \mathrm{SYN}$ assemblies.

\section{Supplementary Information and Extended Data}

Supplementary Information and Extended Data are online available as separate file. 


\section{Acknowledgements}

The authors thank Joris Van Asselbergs and Caroline van Heijningen for their technical assistance and Dr. Zeger Debyser for revising the manuscript. We thank Dr. Johan Hofkens and Charlotte David for the use of the CLSM and Dr Virginie Redeker for assessing the number of atto dyes bound to $\alpha$ SYN by mass spectrometry. The authors thank the Leuven Viral Vector Core (LVVC) for the rAAV vector construction, optimization and production. M.G., R.P., and A.M. are in debt with M. Wijnants and D. Van Dyck for their excellent technical assistance. Research was funded by the FWOVlaanderen (G.0768.10, G.0927.14 and PhD fellowship to WP), the IWT-Vlaanderen (IWT SBO/80020 Neuro-TARGET, SBO/110068 OPTOBRAIN and SBO/130065 MIRIAD), the FP7 RTD projects MEFOPA (HEALTH-2009-241791) and INMiND (HEALTH-F2-2011-278850), the KU Leuven (OT/08/052A, OT/14/120, IMIR PF/10/017), the Agence Nationale de la Recherche (ANR-09-MNPS-013-01 and ANR11-BSV8-021-01), the Centre National de la Recherche Scientifique, a 'Coup d'Elan à la Recherche Française' award from Fondation Bettencourt Schueller, the EC-FP7 (Marie Curie Initial Training Network "NAMASEN", grant n. 264872, the ICT-FET projects "ENLIGHTENMENT" and "BRAINLEAP", grants n. $284801 \&$ 306502), and the

Belgian Science Policy Office (grant n. IUAP-VII/20). The authors declare that there is no actual or potential conflict of interest. R.M. dedicates this work to late professor Paul Cohen.

\section{Author Contributions}

W.P. designed and conducted in vivo experiments. L.B. generated $\alpha \mathrm{SYN}$ assemblies and performed characterization of $\alpha \mathrm{SYN}$ strains. A.V.P. conducted in vivo experiments. C.V.H. was responsible for viral vector production. R.M. co-designed the study. V.B. was responsible for the overall design and coordination of the study. M.G., R.P., and A.M conducted intracellular and extracellular in vitro experiments and analyzed the electrophysiological recordings. W.P., V.B. and R.M. prepared the manuscript.

\section{Competing Financial Interests}

The authors declare to have no competing financial interests. 
*Correspondence to: veerle.baekelandt@med.kuleuven.be and ronald.melki@lebs.cnrsgif.fr

\section{References}

1 Lee, S. J., Desplats, P., Sigurdson, C., Tsigelny, I. \& Masliah, E. Cell-to-cell transmission of non-prion protein aggregates. Nat Rev Neurol 6, 702-706, doi:nrneurol.2010.145 [pii]

10.1038/nrneurol.2010.145 (2010).

2 Goedert, M., Clavaguera, F. \& Tolnay, M. The propagation of prion-like protein inclusions in neurodegenerative diseases. Trends Neurosci 33, 317-325, doi:10.1016/j.tins.2010.04.003 (2010).

3 Guo, J. L. et al. Distinct alpha-Synuclein Strains Differentially Promote Tau Inclusions in Neurons. Cell 154, 103-117, doi:Doi 10.1016/J.Cell.2013.05.057 (2013).

4 Bousset, L. et al. Structural and functional characterization of two alphasynuclein strains. Nature communications 4, 2575, doi:10.1038/ncomms3575 (2013).

5 Li, J. Y. et al. Lewy bodies in grafted neurons in subjects with Parkinson's disease suggest host-to-graft disease propagation. Nat Med 14, 501-503, doi:nm1746 [pii]

$10.1038 / \mathrm{nm} 1746$ (2008).

6 Kordower, J. H., Chu, Y., Hauser, R. A., Freeman, T. B. \& Olanow, C. W. Lewy body-like pathology in long-term embryonic nigral transplants in Parkinson's disease. Nature medicine 14, 504-506, doi:10.1038/nm1747 (2008).

7 Lee, H. J., Bae, E. J. \& Lee, S. J. Extracellular alpha-synuclein-a novel and crucial factor in Lewy body diseases. Nat Rev Neurol 10, 92-98, doi:10.1038/nrneurol.2013.275 (2014).

8 Conway, K. A. et al. Acceleration of oligomerization, not fibrillization, is a shared property of both alpha-synuclein mutations linked to early-onset Parkinson's disease: implications for pathogenesis and therapy. Proc Natl Acad Sci U S A 97, 571-576 (2000).

9 Winner, B. et al. In vivo demonstration that alpha-synuclein oligomers are toxic. P Natl Acad Sci USA 108, 4194-4199, doi:Doi 10.1073/Pnas.1100976108 (2011).

10 Pieri, L., Madiona, K., Bousset, L. \& Melki, R. Fibrillar alpha-synuclein and huntingtin exon 1 assemblies are toxic to the cells. Biophys $J$ 102, 2894-2905, doi:S0006-3495(12)00550-4 [pii]

10.1016/j.bpj.2012.04.050 (2012).

11 Luk, K. C. et al. Pathological alpha-synuclein transmission initiates Parkinsonlike neurodegeneration in nontransgenic mice. Science 338, 949-953, doi:338/6109/949 [pii]

10.1126/science.1227157 (2012). 
12 Sacino, A. N. et al. Induction of CNS alpha-synuclein pathology by fibrillar and non-amyloidogenic recombinant alpha-synuclein. Acta Neuropathol Commun 1, 38, doi:2051-5960-1-38 [pii]

10.1186/2051-5960-1-38 (2013).

13 Ulusoy, A., Decressac, M., Kirik, D. \& Bjorklund, A. Viral vector-mediated overexpression of alpha-synuclein as a progressive model of Parkinson's disease. Prog Brain Res 184, 89-111, doi:S0079-6123(10)84005-1 [pii]

10.1016/S0079-6123(10)84005-1 (2010).

14 Van der Perren, A. et al. Longitudinal follow-up and characterization of a robust rat model for Parkinson's disease based on overexpression of alpha-synuclein with adeno-associated viral vectors. Neurobiol Aging, doi:10.1016/j.neurobiolaging.2014.11.015 (2014).

15 Rey, N. L., Petit, G. H., Bousset, L., Melki, R. \& Brundin, P. Transfer of human alpha-synuclein from the olfactory bulb to interconnected brain regions in mice. Acta Neuropathol 126, 555-573, doi:10.1007/s00401-013-1160-3 (2013).

16 Reyes, J. F. et al. Alpha-synuclein transfers from neurons to oligodendrocytes. Glia, doi:10.1002/glia.22611 (2013).

17 Janezic, S. et al. Deficits in dopaminergic transmission precede neuron loss and dysfunction in a new Parkinson model. Proc Natl Acad Sci U S A 110, E40164025, doi:10.1073/pnas.1309143110 (2013).

18 Milber, J. M. et al. Lewy pathology is not the first sign of degeneration in vulnerable neurons in Parkinson disease. Neurology 79, 2307-2314, doi:10.1212/WNL.0b013e318278fe32 (2012).

19 Kramer, M. L. \& Schulz-Schaeffer, W. J. Presynaptic alpha-synuclein aggregates, not Lewy bodies, cause neurodegeneration in dementia with Lewy bodies. $J$ Neurosci 27, 1405-1410, doi:10.1523/JNEUROSCI.4564-06.2007 (2007).

20 Wilhelm, B. G. et al. Composition of isolated synaptic boutons reveals the amounts of vesicle trafficking proteins. Science 344, 1023-1028, doi:10.1126/science.1252884 (2014).

21 Greten-Harrison, B. et al. alphabetagamma-Synuclein triple knockout mice reveal age-dependent neuronal dysfunction. Proc Natl Acad Sci U S A 107, 1957319578, doi:1005005107 [pii]

10.1073/pnas.1005005107 (2010).

22 Buell, A. K. et al. Solution conditions determine the relative importance of nucleation and growth processes in alpha-synuclein aggregation. Proc Natl Acad Sci U S A 111, 7671-7676, doi:10.1073/pnas.1315346111 (2014).

23 Brundin, P., Melki, R. \& Kopito, R. Prion-like transmission of protein aggregates in neurodegenerative diseases. Nat Rev Mol Cell Bio 11, 301-307, doi:Doi 10.1038/Nrm2873 (2010).

24 Mabbott, N. A. \& MacPherson, G. G. Prions and their lethal journey to the brain. Nat Rev Microbiol 4, 201-211, doi:nrmicro1346 [pii]

10.1038 /nrmicro1346 (2006).

25 Sacino, A. N. et al. Intramuscular injection of alpha-synuclein induces CNS alpha-synuclein pathology and a rapid-onset motor phenotype in transgenic mice. 
Proc Natl Acad Sci U S A 111, 10732-10737, doi:10.1073/pnas.1321785111 (2014).

26 Holmqvist, S. et al. Direct evidence of Parkinson pathology spread from the gastrointestinal tract to the brain in rats. Acta Neuropathol 128, 805-820, doi:10.1007/s00401-014-1343-6 (2014).

27 Redeker, V., Pemberton, S., Bienvenut, W., Bousset, L. \& Melki, R. Identification of protein interfaces between alpha-synuclein, the principal component of Lewy bodies in Parkinson disease, and the molecular chaperones human Hsc70 and the yeast Ssa1p. The Journal of biological chemistry 287, 32630-32639, doi:10.1074/jbc.M112.387530 (2012).

\section{Figure Legends}

Fig. 1. aSYN assemblies spread but only strains cause Lewy-like pathology after injection in rat SNpc. a, Ex vivo macroscopic imaging of atto-550 labeled $\alpha \mathrm{SYN}$ after nigral inoculation (red injection spot) by reconstructing 11 separate excitation spots into a macroscopic 3D map allowing the visualization of $\alpha \mathrm{SYN}$ spread. Representative image of fluorescently labeled oligomeric aSYN distribution after 20 minutes and 7 days is shown. b, Quantification of change in $\alpha \mathrm{SYN}$ fluorescent volume $(\Delta \mathrm{FV})$ between 20 minutes and 7 days as a measure for $\alpha \mathrm{SYN}$ spreading. Oligomers show a significant increase in spreading $(1.78 \pm 0.44$ s.e.m) compared to free dye $(-0.34 \pm 0.06$ s.e.m. $)$ and $\alpha S Y N$ fibrils $(0.40 \pm 0.21$ s.e.m. $)\left({ }^{\#} p<0.01,{ }^{\#} p<0.05\right.$ for one-way ANOVA with Tukey's post-hoc analysis, $\mathrm{n}=3$ ). c, After 4 months Lewy-like pathology is observed in DA neurons after inoculation of $\alpha \mathrm{SYN}$ strains (fibrils and ribbons). d, Schematic overview of LB- and LN-like inclusions in SNpc for ribbons and fibrils. e, LN-like threads and clubshaped inclusions (upper and lower panel, respectively) show abundant inclusions for ribbons quantified in $\mathbf{f}{ }^{(\# \#+} \mathrm{p}<0.001$ for one-way ANOVA with Tukey's post-hoc analysis, $\mathrm{n}=4)$. g, Confocal images with z-axis show phosphorylated inclusions in DA neurons colocalizing with the ubiquitin binding protein $\mathrm{p} 62, \mathbf{h}$, whereas only ribbons result in GCI's in the presence of elevated $\alpha \mathrm{SYN}$ levels after combined rAAV-driven $\alpha \mathrm{SYN}$ overexpression i, further indicated by the oligodendroglial lineage marker Olig2. Scale bars indicate $10 \mu \mathrm{m}$.

Fig. 2. aSYN fibrils are the major toxic species in vivo and cause synaptic impairment. a-b, Stereological quantification of $\mathrm{TH}^{+}$cells and striatal $\mathrm{TH}$ volume shows 
no significant cell or axonal loss after nigral injection of different $\alpha \mathrm{SYN}$ assemblies. c-d, Significant loss of DA cells and projecting axons is observed upon inoculation of fibrils and brain homogenate with rAAV-driven $\alpha \mathrm{SYN}$ overexpression (one-way ANOVA with Tukey's post-hoc analysis versus contralateral side ${ }^{* * *} \mathrm{p}<0.001,{ }^{* *} \mathrm{p}<0.01,{ }^{*} \mathrm{p}<0.05$; or Dunnet's post-hoc analysis versus control condition $\# \mathrm{p}<0.05$; s.e.m, $\mathrm{n}=4$ ). e, Representative image of tyrosine hydroxylase (TH) staining in $\mathrm{SNpc}$ and striatum after co-injection of $\alpha \mathrm{SYN}$ fibrils and rAAV-driven $\alpha \mathrm{SYN}$ overexpression after 4 months. f, Impaired motor behavior after inoculation of $\alpha \mathrm{SYN}$ fibrils $\left({ }^{* *} \mathrm{p}<0.01,{ }^{*} \mathrm{p}<0.05\right.$, onesample t-test for a hypothetical value of $50 \%$ with Bonferroni post-hoc analysis; $\# \mathrm{p}<0.05$ for one-way ANOVA with Tukey's post-hoc analysis; s.e.m, $n=4$ ) and $\mathbf{g}$, increased decline of motor behavior after combined $\alpha \mathrm{SYN}$ assemblies inoculation and rAAVdriven $\alpha$ SYN overexpression. h, Schematic overview indicating neurotoxic effects induced by different $\alpha \mathrm{SYN}$ species with fibrils exhibiting the largest neurotoxic potential. Red plus (+) indicates data obtained upon rAAV-driven $\alpha$ SYN overexpression. Reduced spontaneous collective firing in 4-week old primary rat cortical neurons indicate synaptic impairment. i, Whole-cell patch-clamp intracellular recordings in single neurons revealed a progressive reduction of the rate of spontaneous emission of APs, quantified in $\mathbf{j}$ ( ${ }^{*} \mathrm{p}<0.05,{ }^{*} \mathrm{p}<0.01$, Wilcoxon rank sum test, $\mathrm{n}=32$ cells). $\mathbf{k}$, Representative raster plot of spontaneous AP firing and the effect of fibrils on network-wide spontaneous episodic synchronization of neuronal APs. I, Significant lengthening of the average inter-spike intervals during the episodes of synchronization was observed across increasing concentrations of fibrils and oligomers ( $\mathrm{n}=9$ cultures). No significant changes occurred during the entire incubation time in the total number of microelectrodes detecting spontaneous APs, indicating normal cell viability.

Fig. 3. $\alpha S Y N$ assemblies propagate in a strain-dependent manner. a, sarkosylinsoluble $\alpha \mathrm{SYN}$ is detected in striatum and substantia nigra after striatal inoculation of brain homogenate, oligomers, ribbons and fibrils in combination with rAAV-driven $\alpha S Y N$ overexpression. $\mathbf{b}$, Degradation of sarkosyl-insoluble $\alpha S Y N$ in brain extracts from fibrils and ribbons inoculated animals with rAAV-driven $\alpha \mathrm{SYN}$ overexpression by increasing proteinase $\mathrm{K}$ concentrations. The results reveal that each strain imprints its intrinsic structure to endogenous $\alpha \mathrm{SYN}$. WB analysis was performed using the 
monoclonal anti- $\alpha \mathrm{SYN}$ antibody clone 42 . The data correspond to the mean and associated standard error calculated from 3 independent measurements from 2 biological replicates.

Fig. 4. $\alpha S Y N$ strains cross the BBB after systemic administration. a, $\alpha S Y N$ oligomers, ribbons and fibrils were intravenously injected every two weeks during 4 months in rat tail vein. b, Epifluorescent imaging reveals a fluorescent atto-647 signal originating from the brain region for $\alpha \mathrm{SYN}$ oligomers, ribbons and fibrils 4 months after the initial injection. c, Fluorescent yield is significantly higher for ribbons and oligomers compared to control conditions $\left({ }^{* *} \mathrm{p}<0.01,{ }^{*} \mathrm{p}<0.05\right.$, one-way ANOVA with Dunnet's post-hoc analysis versus control condition). d, Overview panel of huaSYN deposits and microglial activation (Mac1/CD11b staining) in thoracic spinal segment at low and high magnification. e, Quantification of $\alpha \mathrm{SYN}$ density in the spinal cord (cervical, thoracic and lumbosacral segments) shows a strong correlation between $\alpha \mathrm{SYN}$ density and microglial activation. f, Intravenous injection of $\alpha \mathrm{SYN}$ fibrils for 4 months results in $\alpha S Y N$ inclusions in cortical neurons labeled with the neuron-specific marker $\beta$ III-tubulin. g, Confocal analysis of spinal cord reveals huaSYN deposits labeled with the atto-550 and atto-647 markers. Scale bars indicate $20 \mu \mathrm{m}$.

\section{Methods}

\section{Production and purification of recombinant $\alpha$ SYN and $\operatorname{rAAV}-\alpha S Y N$}

Recombinant $\alpha$ SYN production was performed as previously described ${ }^{4}$. Vector production and purification was performed as previously described ${ }^{28}$. The plasmids include the constructs for the AAV2/7 serotype, the AAV transfer plasmid encoding the human A53T mutant $\alpha$-synuclein transgene under the control of the ubiquitous CMVie enhanced synapsin 1 promoter and the pAdvDeltaF6 adenoviral helper plasmid. Real-time PCR analysis was used for genomic copy determination.

\section{Endotoxin detection}

Protein endotoxin detection was performed using LAL Chromogenic Endotoxin Quantitation Kit (Thermofisher ref \#88282) following the manufacturer instruction. 
Briefly, $10 \mu \mathrm{g}$ of $\alpha \mathrm{SYN}$ monomers, fibrils, ribbons and unbound Atto-550 were dissolved in $50 \mu 1$ of endotoxin free water provided in the kit and distributed in 96 well microplate. The LAL reagent was added next and the plate was incubated $10 \mathrm{~min}$ at $37^{\circ} \mathrm{C}$ in a thermomixer (Eppendorf, Germany). The chromogenic reagent was next added and the incubation further extended 6 min. The reaction was arrested with $25 \%$ acetic acid. Absorbance at $405 \mathrm{~nm}$ was measured using a Flexstation microplate reader (FlexStation, Molecular Devices). Protein endotoxin units are presented in Extended Data Figure 1.

rAAV- $\alpha$ SYN vector endotoxin detection was performed using LAL bacterial endotoxin test (Endosafe $\AA$ - PTSTM, Charles River) following the manufacturer instruction. Briefly, rAAV $\alpha S Y N$ vector was loaded in the sample reservoir, mixed with the LAL reagent and combined with the chromogenic substrate. The Optical density was measured and analyzed against an internally achieved standard curve. The measurements were performed in quadruple. Endotoxin units were determined to be 0,0000114 Endotoxin Unit/Injection.

\section{Generation and labeling of $\alpha S Y N$ assemblies}

$\alpha$ SYN fibrils and ribbons were generated as previously described ${ }^{4}$. Oligomeric $\alpha \mathrm{SYN}$ was generated by incubating monomeric $\alpha \mathrm{SYN} 4^{\circ} \mathrm{C}$ for 7 days. Oligomeric $\alpha \mathrm{SYN}$ was separated from monomeric $\alpha \mathrm{SYN}$ by size exclusion chromatography using a Superose ${ }^{\circledR} 6$ HR10/30 column (GE Healthcare) equilibrated in phosphate buffered saline (PBS) buffer.

$\alpha$ SYN assemblies in PBS were labeled by addition of 2 molar excess of the aminoreactive fluorescent dye atto-550 or atto-647 (ATTO-Tech GmbH). Labeling was performed following the manufacturer's recommendations. Unreacted dye or biotin were removed by size exclusion chromatography or three cycles of sedimentation and suspension in PBS for oligomers or fibrils and ribbons, respectively. The amount of incorporated dye was assessed by mass spectrometry. The samples were de-salted (with $5 \%$ acetonitrile, $0.1 \%$ Trifluoroacetic acid (TFA)) and eluted from a C18 reversed-phase Zip-Tip (Millipore, Billerica, MA, USA) in 50\% acetonitrile, 0.1\% TFA. Peptide samples were mixed in a ratio of $1: 5$ to $1: 20(\mathrm{v} / \mathrm{v})$ with sinapinic acid $(10 \mathrm{mg} / \mathrm{mL})$ in $50 \%$ acetonitrile and $0.1 \%$ TFA) and spotted $(0.5 \mu \mathrm{L})$ on a stainless steel MALDI target (Opti- 
TOF; Applied BioSystems). MALDI-TOF-TOF MS spectra were acquired with a MALDI-TOF/TOF 5800 mass spectrometer (Applied Biosystems) using linear mode acquisition. External calibration was performed using unmodified WT $\alpha \mathrm{SYN}$. Acquisition and data analysis were performed using the Data Explorer software from Applied Biosystems.

The nature of all $\alpha \mathrm{SYN}$ assemblies used was routinely assessed using a Jeol 1400 (Jeol Ltd., Peabody, MA) Transmission Electron Microscope (TEM) after adsorption of the samples onto carbon-coated 200-mesh grids and negative staining with 1\% uranyl acetate. The images were acquired with a Gatan Orius CCD camera (Gatan).

\section{Stereotactic injections}

All animal experiments were carried out in accordance with the European Communities Council Directive of 24 November 1986 (86/609/EEC) and approved by the Bioethical Committee of the KU Leuven (Belgium). Young adult female Wistar rats (Janvier, France) weighing about 200-250 g were housed under a normal $12 \mathrm{~h}$ light/dark cycle with free access to pelleted food and tap water. All surgical procedures were performed using aseptic techniques and ketamine $\left(60 \mathrm{mg} / \mathrm{kg}\right.$ ip., Ketalar ${ }^{\circledR}$, Pfizer, Belgium) and medetomidine $\left(0.4 \mathrm{mg} / \mathrm{kg}\right.$, Dormitor ${ }^{\circledR}$, Pfizer $)$ anaesthesia. Following anaesthesia the rodents were placed in a stereotactic head frame (Stoelting, IL, USA). Injections were performed with a 30 -gauge needle and a $10-\mu 1$ Hamilton syringe. After making a midline incision of the scalp, a burr hole was drilled in the appropriate location for the SN (single injections) or striatum (triple injections) at the right site of the skull. Stereotactic coordinates, dose and concentration of $\alpha \mathrm{SYN}$ protein and $\mathrm{rAAV}-\alpha \mathrm{SYN}$ vectors are described in Extended data Table 1. Brain homogenate was extracted from 18 month old Thy-1 A30P transgenic mice ${ }^{29}$ as described previously ${ }^{30}$ adjusted to a total amount of 10 $\mu \mathrm{g}$. Supplemented viral titers were diluted to $3.0 \mathrm{E}+10 \mathrm{GC} / \mathrm{ml}$ A53T $\alpha \mathrm{SYN}$ rAAV2/7.

\section{Fluorescent imaging}

Epifluorescent images and fluorescent tomography were performed using the IVIS Spectrum in vivo imaging system (Caliper, PerkinElmer, version 4.3.1). Anesthesia was induced in an induction chamber with 3\% isoflurane in 100\% oxygen at a flow rate of 
$1 \mathrm{~L} / \mathrm{min}$ and maintained in the IVIS with a $1.5 \%$ mixture at $0.5 \mathrm{~L} / \mathrm{min}$. Rats were injected with $500 \mu 1$ saline supplemented with $10 \mu \mathrm{g}$ of fluorescently-labeled $\alpha \mathrm{SYN}$ protein intravenously in the tail vein. Subsequently, they were placed in the prone position in the IVIS and epifluorescent images of atto-647 $\alpha$ SYN labeled proteins were acquired at the final time point. Atto-550 $\alpha \mathrm{SYN}$ labeled proteins could not be imaged due to high autofluorescent background levels in live animals. Each frame depicts the fluorescent image as a pseudocolor image superimposed on the gray-scale photographic image. The data are reported on a logarithmic scale as the fluorescent yield or fluorescence emission radiance per incident excitation intensity $\left(\mathrm{p} / \mathrm{sec} / \mathrm{cm}^{2} / \mathrm{sr} / \mu \mathrm{W} / \mathrm{cm}^{2}\right)$ from a $2 \mathrm{~cm}^{2}$ circular region of interest around the head.

Fluorescent tomography ex vivo images of $\alpha$ SYN spreading were acquired by merging 11 distinct and predefined transillumination excitation sources of perfused and fixed rat brain. A small illumination spot is scanned over a predefined grid resulting in distinct illumination foci. The excitation source was positioned below the brain with the delivery of a concentrated excitation light beam and observed by a detector located above the object. Superimposing surface topography and fluorescent signal results in fluorescent tomography providing a $3 \mathrm{D}$ reconstruction of atto-550-tagged $\alpha \mathrm{SYN}$ fluorescent volume $(\mathrm{FV})$. Emitted fluorescent light propagation is calculated through diffusion approximation allowing the reconstruction of the fluorescent source in 3D. 3D reconstruction is presented as voxels with fluorescent intensity profile for each condition and is mapped to a $3 \mathrm{D}$ brain atlas. Fluorescence yield is expressed in units of $\left[\mathrm{pmolxM}^{-1} \mathrm{xcm}^{-1}\right]$. A threshold \% value was set to keep the minimum count of pixels above 200 to exclude background pixels from the reconstruction. The accuracy of reconstruction was checked for all samples by comparing the measured light diffusion pattern with the simulated light diffusion pattern. If the $\%$ error between these two has a near-zero $\%$ difference, the reconstruction was considered successful and used for spatial measurements.

\section{Behavioral testing}

The cylinder test was used to measure asymmetry in spontaneous forelimb use. Contacts made by each forepaw with the wall of $20 \mathrm{~cm}$-wide clear glass cylinder were scored from the videotapes by an observer blinded to the animal's identity. Between 20 and 30 wall 
touches per animal (contacts with fully extended digits executed with the forelimb ipsilateral and contralateral to the lesion) were counted. The number of impaired forelimb contacts was expressed as a percentage of total forelimb contacts. Non-lesioned control rats should score around $50 \%$ in this test. No habituation of the animals to the testing cylinder was allowed before video recording. An investigator blind to different groups performed all the analyses.

\section{Histology}

Rats were sacrificed with an overdose of sodium pentobarbital (60mg/kg, i.p., Nembutal ${ }^{\circledR}$, Ceva Santé, Belgium) followed by intracardial perfusion with $4 \%$ paraformaldehyde in PBS. After postfixation overnight, $50 \mu \mathrm{m}$ thick coronal brain sections were made with a vibrating microtome (HM 650V, Microm, Germany). IHC was performed as previously described ${ }^{31}$ using antibodies summarized in Extended data Table 3. For spinal cord staining, commercial TNB blocking buffer (Perkin Elmer) was added to all incubation steps to minimize background staining in $50 \mu \mathrm{m}$ vibratome sections.

For fluorescent double or triple staining, sections were rinsed three times in PBS and incubated overnight in the dark in PBS- $0.1 \%$ Triton X-100 and $0.1 \%$ sodium azide, $10 \%$ donkey or goat serum and antibodies summarized in Extended data Table 3. After rinsing, the sections were incubated in the dark for $2 \mathrm{~h}$ in fluorochrome- conjugated secondary antibodies. After being rinsed and mounted, the sections were cover-slipped with Mowiol. Fluorescent staining was visualized by confocal microscopy (Fluoview 1000, Olympus). Reconstructed 3D images from Z-stack tiles were imaged using an Olympus confocal laser-scanning microscope and rendered into the complete neuronal reconstructions by tiling the entire set of z-stacks using Imaris software.

\section{Stereological quantification}

The number of TH-positive cells and $\alpha \mathrm{SYN}$ positive cells in the SN was determined by stereological measurements using the Optical fractionator method in a computerized system as described before ${ }^{32}$ (StereoInvestigator; MicroBright-Field, Magdeburg, Germany). Every fifth section throughout the entire SN was analyzed, with a total of seven sections for each animal. The coefficients of error, calculated according to the 
procedure of Schmitz and Hof as estimates of precision ${ }^{33}$ varied between 0.05 and 0.10. The volume of $\alpha \mathrm{SYN}$ expression in the brain and the SN was determined by the Cavalieri method. Every fifth section covering the entire extent of the SN, with a total of seven sections for each animal, was included in the counting procedure. The coefficients of error varied between 0.05 and 0.14 . We quantified both the injected and non-injected SN (internal control), no cell loss was observed in the non-injected side. An investigator blind to different groups performed all the analyses.

\section{Sarkosyl extraction}

Unfixed brains were dissected to isolate $\mathrm{SNpc}$ and striatum from both hemispheres and frozen in liquid nitrogen. Brain samples were weighted and homogenized at 10\% (W/V) in PBS buffer supplemented with Phosphatase inhibitor cocktail (sigma P5726 and P0044) and protease inhibitors (Roche Complete EDTA free). Sarkosyl was added to 1\%. $250 \mu \mathrm{l}$ were spun at $1000 \mathrm{~g}$ for 30 minutes to remove cell debris. The supernatants $(200 \mu \mathrm{l})$ were further spun at $200,000 \mathrm{~g}$ for 60 minutes at $20^{\circ} \mathrm{C}$ in an Optima MAX-XP Ultracentrifuge (Beckman). The pellets were washed with PBS-1\% Sarkosyl, and resuspended in $200 \mu 1$ of PBS buffer.

\section{Proteinase K digestion, SDS-, native-PAGE and western blot analysis}

Sarkosyl insoluble fractions containing $\alpha \mathrm{SYN}$ were incubated at $25^{\circ} \mathrm{C}$ with Proteinase $\mathrm{K}$ $(0.001,0.01,0.1,1 \mu \mathrm{g} / \mathrm{ml})$ (Roche) for $30 \mathrm{~min}$. Aliquots were mixed $\mathrm{V} / \mathrm{V}$ with denaturing buffer $(50 \mathrm{mM}$ Tris- $\mathrm{HCl}, \mathrm{pH} 6.8,4 \%$ SDS, 2\% 3 -mercaptoethanol, 12\% glycerol and $0.01 \%$ bromophenol blue) and heated at $90^{\circ} \mathrm{C}$ to arrest immediately the cleavage reaction. After incubation for $10 \mathrm{~min}$ at $90^{\circ} \mathrm{C}$, the samples were processed to monitor the time course of $\alpha$ SYN cleavage by SDS-PAGE (15\% polyacrylamide gels) followed by western blotting and detection with Clone 42 monoclonal antibody (BD Bioscience ref. 610787) or anti-phospho-Ser 129 aSYN antibody. For native-PAGE, sarkosyl insoluble fractions subjected or not to PK treatment were supplemented with Triton X-100 (0.2 \%, v/v) Bmercaptoethanol (2\%), glycerol (12\%) and bromophenol blue $(0.01 \%)$ and loaded on $15 \%$ native-PAGE. The samples were transferred to nitrocellulose membranes and probed with D37A6 or Syn 211 anti- rodent and human $\alpha$ SYN antibodies (Extended data 
Table 3).

\section{Electrophysiology and MEA recordings}

Primary cortical cultures were obtained from postnatal Wistar rats, employing standard methods ${ }^{34}$. Briefly, after mechanical and enzymatic dissociation of the cerebral cortices of postnatal day 0 (P0) pups, cells were plated at a final density of $\sim 1.500 \mathrm{~mm}^{-2}$ on glass coverslips and of $6{ }^{5} 500 \mathrm{~mm}^{-2}$ on commercial microelectrode arrays (MEAs; Multichannel Systems GmBH, Reutlingen, Germany). Individual MEAs incorporated 59 substrate microelectrodes made of Indium Titanium Nitrate, featuring a diameter of $30 \mu \mathrm{m}$ and an electrical impedance of $\sim 100 \mathrm{kOhm}$ at $1 \mathrm{kHz}$. The inter-electrode spacing was $200 \mu \mathrm{m}$, and the microelectrodes arranged in a regular $8 \times 8$ layout that covered an area of $\sim 2,56 \mathrm{~mm}^{2}$. MEAs and coverslips were coated overnight with PolyEthyleneImine $0.1 \%(\mathrm{wt} / \mathrm{vol})$ in millQ water at room temperature, prior to cell seeding. Seeded MEAs and coverslips were maintained under sterile conditions at $37^{\circ} \mathrm{C}$ under $5 \% \mathrm{CO} 2$ atmosphere with $100 \%$ R.H. for several weeks.

Patch-clamp intracellular recordings were performed 22-23 days after plating, from neurons cultured under control conditions (i.e., $1 \mu \mathrm{M}$ Bovine Serum Albumin) or incubated for 3-4 days with $1 \mu \mathrm{M} \alpha \mathrm{SYN}$ (i.e., as ribbons, fibrils, oligomers). Patch-clamp recordings were made from the cell somata under the whole-cell configuration using an Axon Multiclamp 700B Amplifier (Molecular Devices LLC, Sunnyvale, CA, US), employing both current-clamp and voltage-clamp modes. Patch electrodes were pulled from thick-walled borosilicate glass capillaries (1BF150, World Precision Instruments, Hitchin, UK) by a horizontal puller (P97, Sutter, Novato, US) to a resistance of 6-7M 2 . Electrodes were filled with an intracellular solution containing (in $\mathrm{mM}$ ): $135 \mathrm{~K}$ gluconate, $10 \mathrm{KCl}$, 10 HEPES, 0.2 EGTA, 4 Mg-ATP, $0.4 \mathrm{Na}_{3} \mathrm{GTP}$, and $14 \mathrm{Na}_{2}$ phosphocreatine $(\mathrm{pH} \mathrm{7.3,} \mathrm{titrated} \mathrm{with} \mathrm{KOH})$. All recordings were obtained at $34^{\circ} \mathrm{C}$ and replacing the culture medium by an artificial cerebro-spinal fluid, constantly perfused at a rate of $1 \mathrm{ml} / \mathrm{min}$ and containing (in $\mathrm{mM}$ ): $145 \mathrm{NaCl}, 4 \mathrm{KCl}, 2$ Na-pyruvate, 5 Hepes, 5 glucose, $2 \mathrm{CaCl}_{2}$, and $1 \mathrm{MgCl}_{2}$ (pH adjusted to 7.4 with $\mathrm{NaOH}$ ). Voltage and current traces were sampled at $15 \mathrm{kHz}, \mathrm{A} / \mathrm{D}$ converted at 16 bits, and stored on a PC employing the software $\mathrm{LCG}^{35}$. Data were analyzed off-line, employing custom scripts written in 
MATLAB (The MathWorks, Natick, US) or Excel (Microsoft, USA).

Extracellular recordings experiments were performed 15-20 days after plating, and continued until day 28, from neurons cultured over MEAs under control conditions (i.e., $1 \mu \mathrm{M}$ Bovine Serum Albumin) or incubated for up to 10 days with $1 \mu \mathrm{M} \alpha \mathrm{SYN}$ (i.e., as ribbons, fibrils, oligomers). Recordings were performed by a MEA1060BC amplifier, (Multichannel Systems GmBH, Reutlingen, Germany) inside an electronic-friendly incubator, maintaining $37^{\circ} \mathrm{C}, 5 \% \mathrm{CO}_{2}$, and $100 \%$ R.H. This allowed us to monitor simultaneously, chronically, and non-invasively the neuronal electrical activity from up to 60 locations within the very same culture. MEA recordings were analyzed off-line by QSpikeTools $^{36}$. Briefly, the extracellular electric fields were monitored from the 59 independent electrodes in each MEAs were sampled at $25 \mathrm{kHz} /$ channel, 1200x amplified, bandpass-filtered $(200-3000 \mathrm{~Hz})$, and digitally recorded for $30 \mathrm{~min}$ every day, up to 6 days following the bath application of $\alpha \mathrm{SYN}$, dissolved in culture medium with distinct concentrations (i.e., 0.1, 0.5, and $1 \mathrm{mM}$ ). Simple spike-sorting, based on spikeshape peak polarity, was performed. Epochs of spontaneous synchronized firing across the MEAs electrodes were identified over $25 \mathrm{~ms}$ bins by standard procedures ${ }^{36}$, and their features were extracted from each recording session.

\section{Statistical analysis}

Results are expressed as means \pm standard error of the mean. Normality of data was assessed with the Lilliefors test. Statistical significance was assessed using one-way ANOVA followed by Dunnet's or Tukey's post-hoc test and column statistics followed by Bonferroni's post-hoc test. Null hypothesis was rejected if the $\mathrm{P}$ value was below 0.5 . Statistical significance level was set as follows: * if $\mathrm{P}<0.05, * *$ if $\mathrm{P}<0.01, * * *$ if $\mathrm{P}<0.001$. In case of improper fitting of data by a normal distribution, median and interquartile range were used for data representation, and Wilcoxon rank sum test was used for analysis.

\section{References}

4 Bousset, L. et al. Structural and functional characterization of two alphasynuclein strains. Nature communications 4, 2575, doi:10.1038/ncomms3575 (2013). 
28 Van der Perren, A. et al. Efficient and stable transduction of dopaminergic neurons in rat substantia nigra by rAAV 2/1, 2/2, 2/5, 2/6.2, 2/7, 2/8 and 2/9.

Gene Ther 18, 517-527, doi:gt2010179 [pii]

10.1038/gt.2010.179 (2011).

29 Kahle, P. J. et al. Selective insolubility of alpha-Synuclein in human Lewy body diseases is recapitulated in a transgenic mouse model. Am J Pathol 159, 22152225, doi:Doi 10.1016/S0002-9440(10)63072-6 (2001).

30 Luk, K. C. et al. Intracerebral inoculation of pathological alpha-synuclein initiates a rapidly progressive neurodegenerative alpha-synucleinopathy in mice. $J$ Exp Med 209, 975-986, doi:jem.20112457 [pii]

10.1084/jem.20112457 (2012).

31 Oliveras-Salva, M. et al. rAAV2/7 vector-mediated overexpression of alphasynuclein in mouse substantia nigra induces protein aggregation and progressive dose-dependent neurodegeneration. Mol Neurodegener 8, doi:Artn 44

Doi 10.1186/1750-1326-8-44 (2013).

32 Baekelandt, V. et al. Characterization of lentiviral vector-mediated gene transfer in adult mouse brain. Hum Gene Ther 13, 841-853, doi:10.1089/10430340252899019 (2002).

33 Schmitz, C. \& Hof, P. R. Design-based stereology in neuroscience. Neuroscience 130, 813-831 (2005).

34 Reinartz, S., Biro, I., Gal, A., Giugliano, M. \& Marom, S. Synaptic dynamics contribute to long-term single neuron response fluctuations. Frontiers in neural circuits 8, 71, doi:10.3389/fncir.2014.00071 (2014).

35 Linaro, D., Couto, J. \& Giugliano, M. Command-line cellular electrophysiology for conventional and real-time closed-loop experiments. Journal of neuroscience methods 230, 5-19, doi:10.1016/j.jneumeth.2014.04.003 (2014).

36 Mahmud, M., Pulizzi, R., Vasilaki, E. \& Giugliano, M. QSpike tools: a generic framework for parallel batch preprocessing of extracellular neuronal signals recorded by substrate microelectrode arrays. Frontiers in neuroinformatics $\mathbf{8}, 26$, doi:10.3389/fninf.2014.00026 (2014).

\section{Extended Data Figure Legends}

Extended Data Figure 1. Characterization of $\alpha \mathrm{SYN}$ assemblies. Electron micrographs of $\alpha \mathrm{SYN}$ a, monomers $\mathbf{b}$, oligomers $\mathbf{c}$, fibrils and $\mathbf{d}$, ribbons used throughout this study. The scale bar represents $200 \mathrm{~nm}$. e, Quantification of endotoxin amounts in different recombinant $\alpha$ SYN preparations used throughout this study. Endotoxin levels were below 0,05 units/injection for all condition. Mass spectrometry analysis of covalently labeled fluorescent WT $\alpha$ SYN by MALDI-TOF f, unlabelled WT $\alpha$ SYN (theoretical Mass 14460.1 Da) g, atto-550 WT $\alpha \mathrm{SYN}$ (average molecular ratio 1 atto molecule per $\alpha$-syn molecule) and $\mathbf{h}$, atto- $647 \mathrm{WT} \alpha \mathrm{SYN}$ (average molecular ratio 1 atto molecule per $\alpha \mathrm{SYN}$ 
molecule).

\section{Extended Data Figure 2. Transsynaptic spreading of fluorescently labeled $\alpha$ SYN in} rat striatum. $\alpha \mathrm{SYN}$ assemblies were injected in rat SNpc (red injection spot) and were allowed to spread for 7 days to the striatum. Sagittal and coronal views are depicted in schematic figure a and $\mathbf{b}$ respectively. Representative confocal images of $\alpha \mathrm{SYN}$ spreading in $\mathbf{c}$, dopaminergic neurons and $\mathbf{d}$, striatal dopaminergic axons for $\alpha \mathrm{SYN}$ oligomers, ribbons and fibrils after 7 days. Reconstructed images from Z-stack tiles of the dopaminergic neurons and axons in striatum were rendered into complete axonal reconstructions by tiling the entire set of z-stacks. Scale bar is $20 \mu \mathrm{m}$. The rectangle identifying Atto-550-tagged $\alpha \mathrm{SYN}$ is shown at a higher magnification in upper right corner of sections in lower panels. e, Representative confocal image of recombinant oligomeric Atto-550 labeled $\alpha \mathrm{SYN}$ uptake in medium spiny neurons (MSN) after injection in SNpc. White arrows indicate positive inclusions. f, Quantification of fluorescently labeled $\alpha$ SYN DARPP- $32^{+}$MSN. Uptake is measured after 1 and 7 days for oligomers ( $\left.\mathrm{n}_{1 \text { day }}=1674, \mathrm{n}_{7_{\text {days }}}=2118, \mathrm{n}_{\text {animals }}=3\right)$, ribbons $\left(\mathrm{n}_{1 \text { day }}=2152, \mathrm{n}_{7 \text { days }}=1968\right.$, $\left.\mathrm{n}_{\text {animals }}=3\right)$ and fibrils $\left(\mathrm{n}_{1 \text { day }}=1223, \mathrm{n}_{7 \text { days }}=1486, \mathrm{n}_{\text {animals }}=3\right)$. Oligomers show increased

uptake rates compared to ribbons and fibrils $\left({ }^{* * *} \mathrm{p}<0.001,{ }^{* *} \mathrm{p}<0.01\right.$, one-way ANOVA with Dunnet's multiple comparison test for 7 day time point) and increases over time ("\#\#p $<0.001$, one-way ANOVA with Tukey's multiple comparison test). Error bars indicate s.e.m., scale bar indicates $20 \mu \mathrm{m}$. g, Correlation of spreading volume and transsynaptic spread at 7 days. Oligomers show the highest spreading capacity compared to fibrils and ribbons using two independent techniques and experiments.

Extended Data Figure 3. Histopathological hallmarks after nigral inoculation of $\boldsymbol{\alpha S Y N}$ strains. Immunofluorescent staining in substantia nigra shows a, granular cytoplasmic b, intracytoplasmic and $\mathbf{c}$, nuclear P $\alpha \mathrm{SYN}$ inclusions in dopaminergic neurons. Reconstructed images from Z-stack tiles were rendered into complete reconstructions by tiling the entire set of z-stacks. Scale bar indicates $20 \mu \mathrm{m}$.

Extended Data Figure 4. Phosphorylation pattern of different $\alpha \mathrm{SYN}$ assemblies upon rAAV-driven $\alpha S Y N$ overexpression. a, Inoculation of ribbons and fibrils result in 
increased amount of phosphorylated $\alpha \mathrm{SYN}$ cells in SN. b, Animals where $\alpha \mathrm{SYN}$ overexpression is rAAV-driven yield a total of $12426 \pm 1288$ cells with phosphorylated $\alpha S Y N$ (s.e.m, $n=4$ ). A significant increase of cells with phosphorylated $\alpha S Y N$ is observed upon injection of ribbons and fibrils, to 22254 cells \pm 2800 (s.e.m, $n=4$ ) and 19690 cells \pm 1803 (s.e.m, $\mathrm{n}=4$ ), respectively ( ${ }^{* *} \mathrm{p}<0.01,{ }^{*} \mathrm{p}<0.05$, one-way ANOVA with Dunnet's multiple comparison test versus control, $" \mathrm{p}<0.05$, one-way ANOVA with Tukey's multiple comparison test).

\section{Extended Data Figure 5. Intracellular and extracellular electrophysiological} recordings of early effects of $\alpha S Y N$ assemblies on the electrical properties of single neurons. Rat primary cortical neurons, cultured ex vivo for over 4 weeks, displayed no significant alterations of the intrinsic electrical phenotype after exposure for 3-4 days to $1 \mu \mathrm{M}$ of $\alpha \mathrm{SYN}$ assemblies. This was characterized by means of intracellular recordings, and analyzed in current-clamp in terms of membrane passive properties $(n=32$ cells), $\mathbf{a}$, as membrane time constant, $\mathbf{b}$, membrane apparent input resistance, $\mathbf{c}$, resting membrane potential $\mathbf{e}$, as well as of evoked excitable responses $(n=30$ cells $)$. By the same technique, the amplitude of $\mathbf{d}$, excitatory spontaneous post-synaptic currents (PSCs) revealed a significant downregulation, recorded under voltage-clamp $(n=32$ cells $)$.

\section{Extended Data Figure 6. $\alpha$ SYN strains propagate after intracerebral inoculation. a,} Preformed $\alpha$ SYN fibrils and ribbons resist 1\% sarkosyl treatment and are pelleted upon sedimentation at $200,000 \mathrm{~g}$ for $60 \mathrm{~min}$ at $25^{\circ} \mathrm{C}$ in vitro. (T, total; S, Soluble; P, Pellet) b, Fibrils resist $1 \%$ sarkosyl treatment and persist more after striatal injections than ribbons or oligomers. Sarkosyl-insoluble $\alpha \mathrm{SYN}$ from animals inoculated fibrils or ribbons is phosphorylated. Mean and associated standard error was calculated from 3 independent quantifications of the intensities made using Image $J$ on samples from two independent experiments.

Extended Data Figure 7. $\alpha$ SYN strains recruit rodent $\alpha$ SYN. Seeding of endogenous $\alpha S Y N$ in rat brain by exogenous human ribbons, fibrils or diseased rat brain homogenates. PBS was inoculated to the control animal. The nature of $\alpha \mathrm{SYN}$ assemblies was assessed by Western blot following Native PAGE separation prior (-) or after $(+)$ 
proteinase K $(76 \mathrm{ng} / \mu \mathrm{l})$ treatment. a, Staining with rat specific antibody (D37A6) detects endogenous $\alpha \mathrm{SYN}$ and, b, staining with human specific antibody (Syn211) detects exogenous injected $\alpha \mathrm{SYN}$ on a duplicate blot. Arrows indicate fibrillar $\alpha \mathrm{SYN}$ in the bottom of the gel well. The non specific band $(*)$ is recognized by the secondary antibody. Representative image from 3 independent experiments is shown

\section{Extended Data Figure 8. aSYN fibrils persist months after intracerebral} inoculation. a, $\alpha \mathrm{SYN}$ assemblies were injected in rat $\mathrm{SNpc}$ (red injection spot) and assessed 4 months later in striatum. b. Immunohistochemical analysis of rat striatum reveals clear human $\alpha \mathrm{SYN}^{+}$inclusions colocalizing with dopaminergic axons. Representative image of fibrils is shown with detailed image in right upper corner. Right panel shows pseudocolor image of only human $\alpha \mathrm{SYN}$. Scale bar indicates $30 \mu \mathrm{m}$. c, Quantification of different $\alpha$ SYN species in rat striatum by means of total fluorescent units. Fibrils appear to persist to a much higher extent compared to ribbons ( ${ }^{\# \#} \mathrm{p}<0.001$, one-way ANOVA with Tukey's multiple comparison test, $\mathrm{n}=4$ ). Oligomers and brain homogenate show very low immunofluorescent staining for human specific $\alpha \mathrm{SYN}$. d, Fibrils appear as axonal inclusions and are abundantly present in striatum in contrast to ribbons after nigral injection. Oligomers and brain homogenate are not detected using the same human specific antibody Syn211. Scale bar indicates $40 \mu \mathrm{m}$.

\section{Extended Data Figure 9. aSYN ribbons and fibrils spread across the CNS after} intravenous administration. Overview of huaSYN (Syn211) inclusions in different areas of the CNS after intravenous injection of $\alpha \mathrm{SYN}$ oligomers, ribbons and fibrils. Scale bar indicates $50 \mu \mathrm{m}$.

\section{Extended Data Figure 10. Intravenous injection of aSYN strains leads to increased microglial response and distinct histopathological hallmarks in the spinal cord.} Quantification of huaSYN density and Mac1/CD11b microglial response following intravenous administration of a saline solution or $\alpha \mathrm{SYN}$ oligomers, ribbons or fibrils in ab, cervical, $\mathbf{c}-\mathbf{d}$, thoracic and e-f, lumbosacral segments $\left({ }^{* *} \mathrm{p}<0.05\right.$, one-way ANOVA with Dunnet's multiple comparison test versus control, ${ }^{\# \#}<<0.001, " \# p<0.01, ", p<0.05$, one-way ANOVA with Tukey's multiple comparison test, $\mathrm{n}=4$ ). Error bars indicate 
s.e.m., white boxes and grey boxes indicate 60 and 120 days after intravenous injections, respectively. g, Immunohistochemical staining for growth associated protein 43 (GAP43), an early injury marker, shows a filamentous distribution for $\alpha \mathrm{SYN}$ ribbons and cellular distribution for $\alpha \mathrm{SYN}$ fibrils (white arrow heads). Scale bar indicates $50 \mu \mathrm{m}$. 
c

e
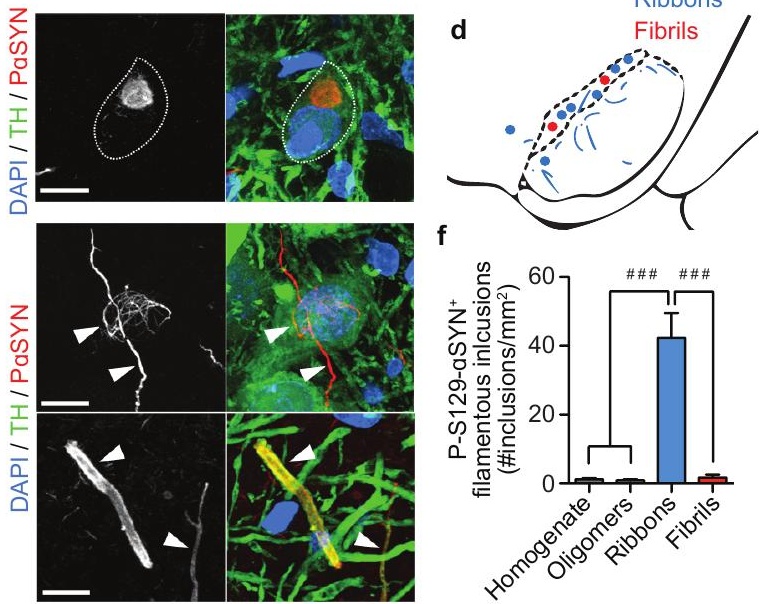

g

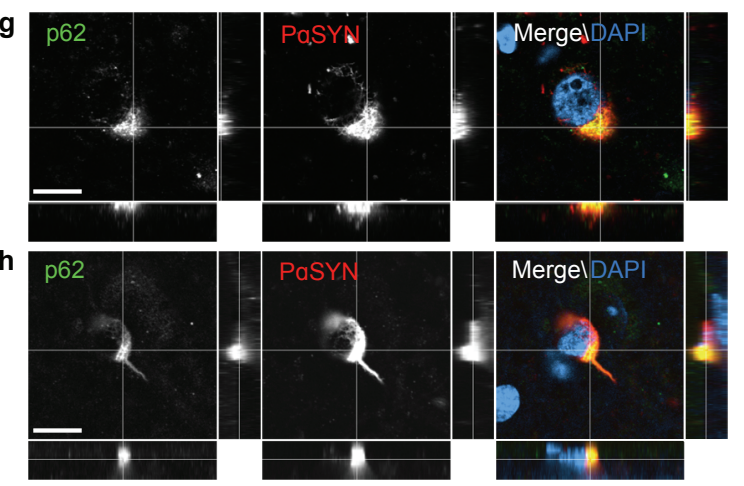

h
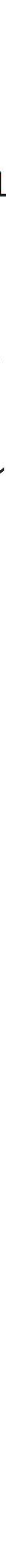

f

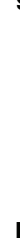

Merge

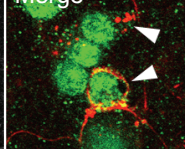




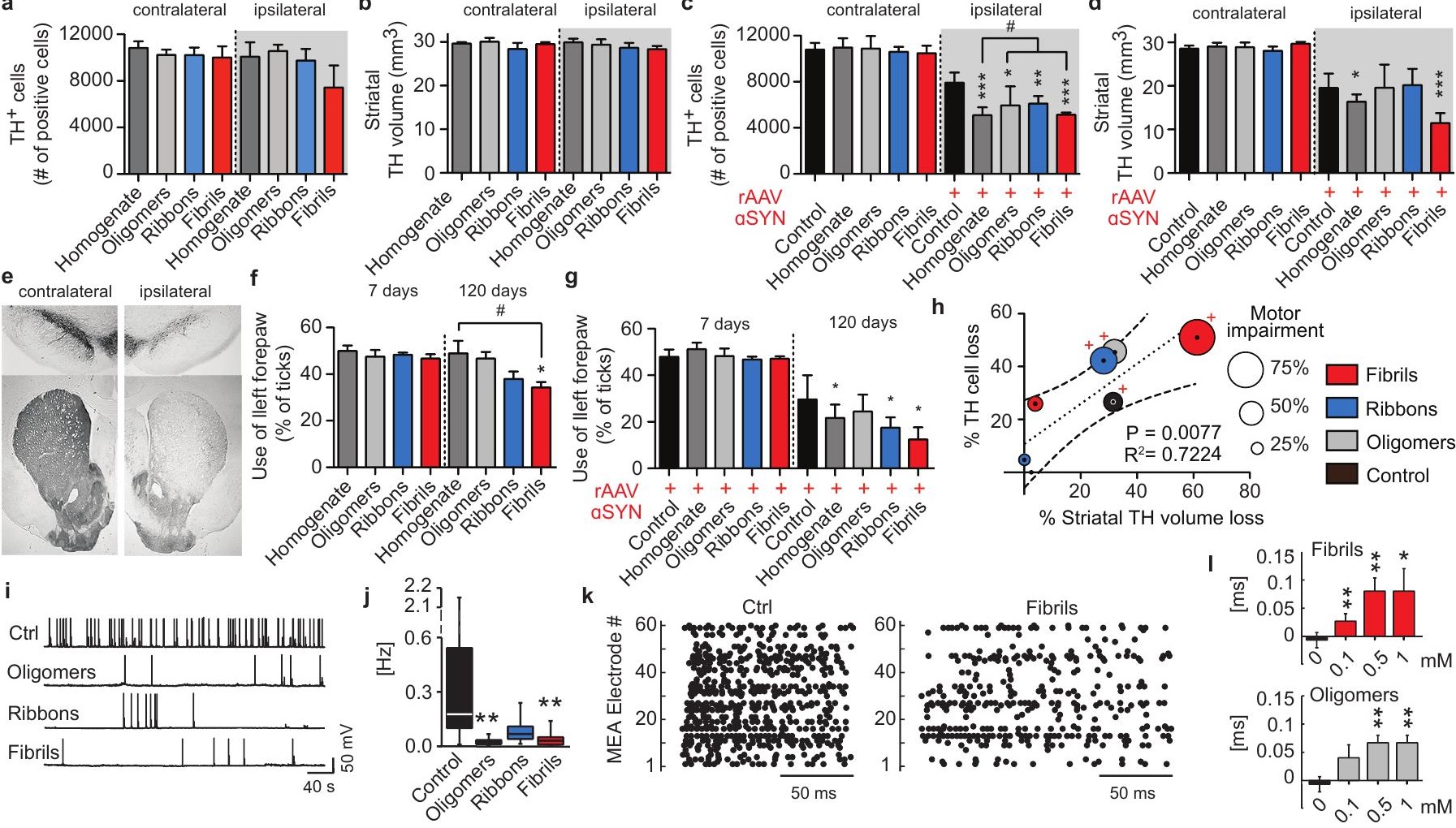


a

Striatum

Substantia Nigra

b
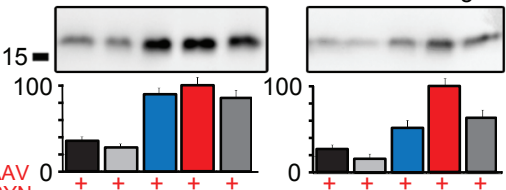

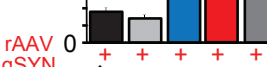

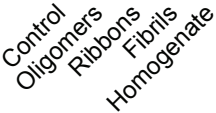

PK

Fibrils

Ribbons

$\mu \mathrm{g} / \mathrm{ml}$

$15=$

100

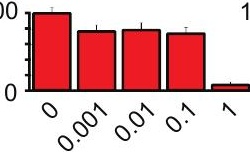

100
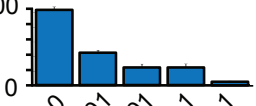

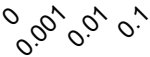




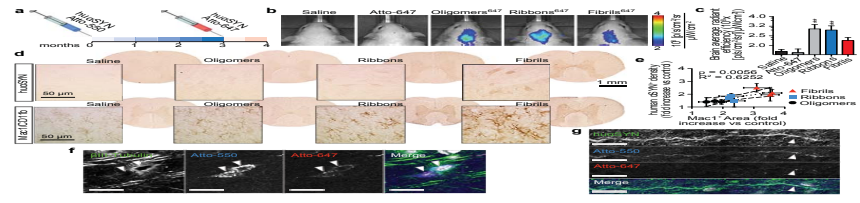




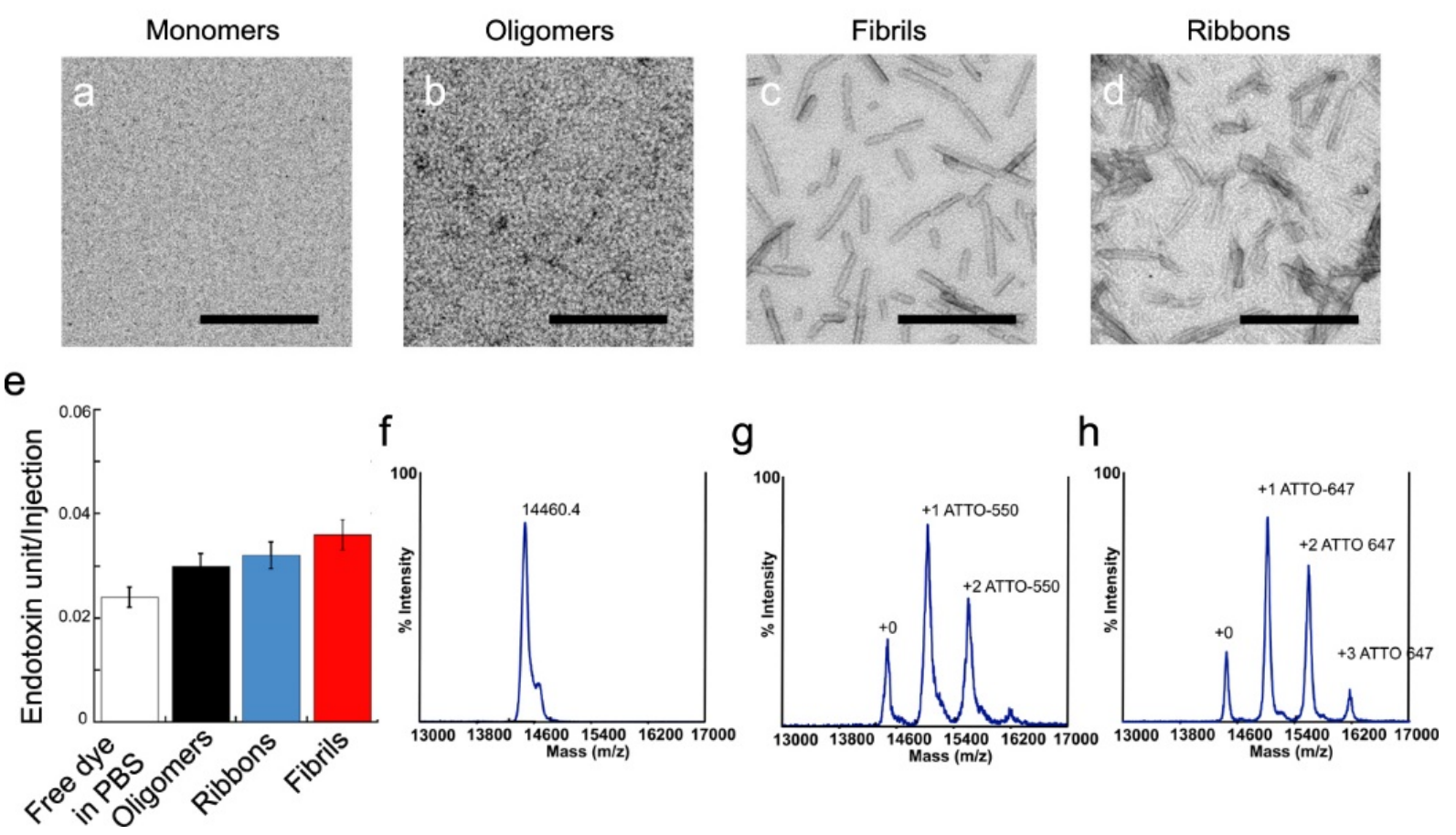

Extended Data Figure 1: Characterization of alpha-SYN assemblies 


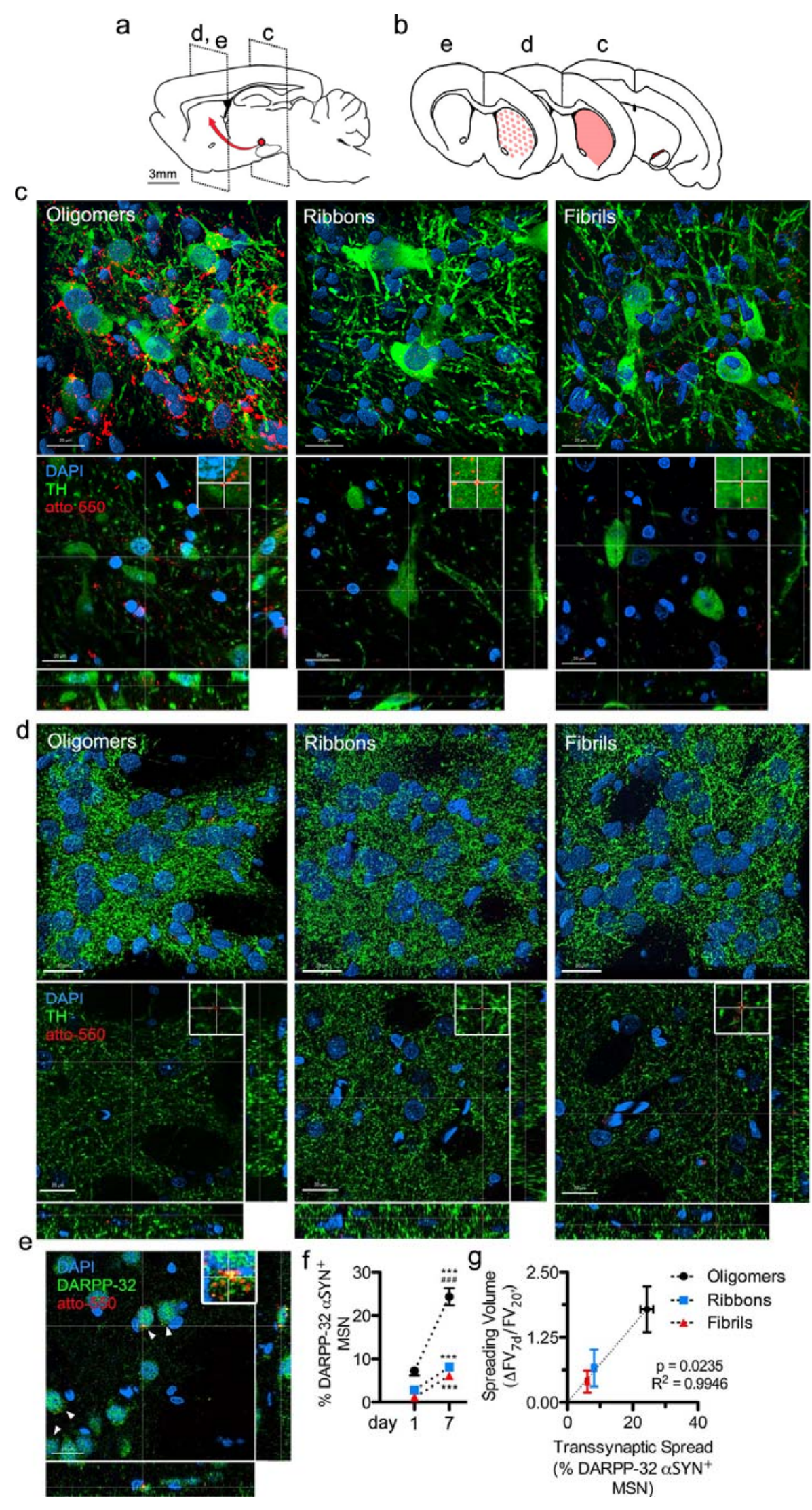

Extended Data Figure 2: Alpha-SYN cellular pre- and postsynaptic spread 

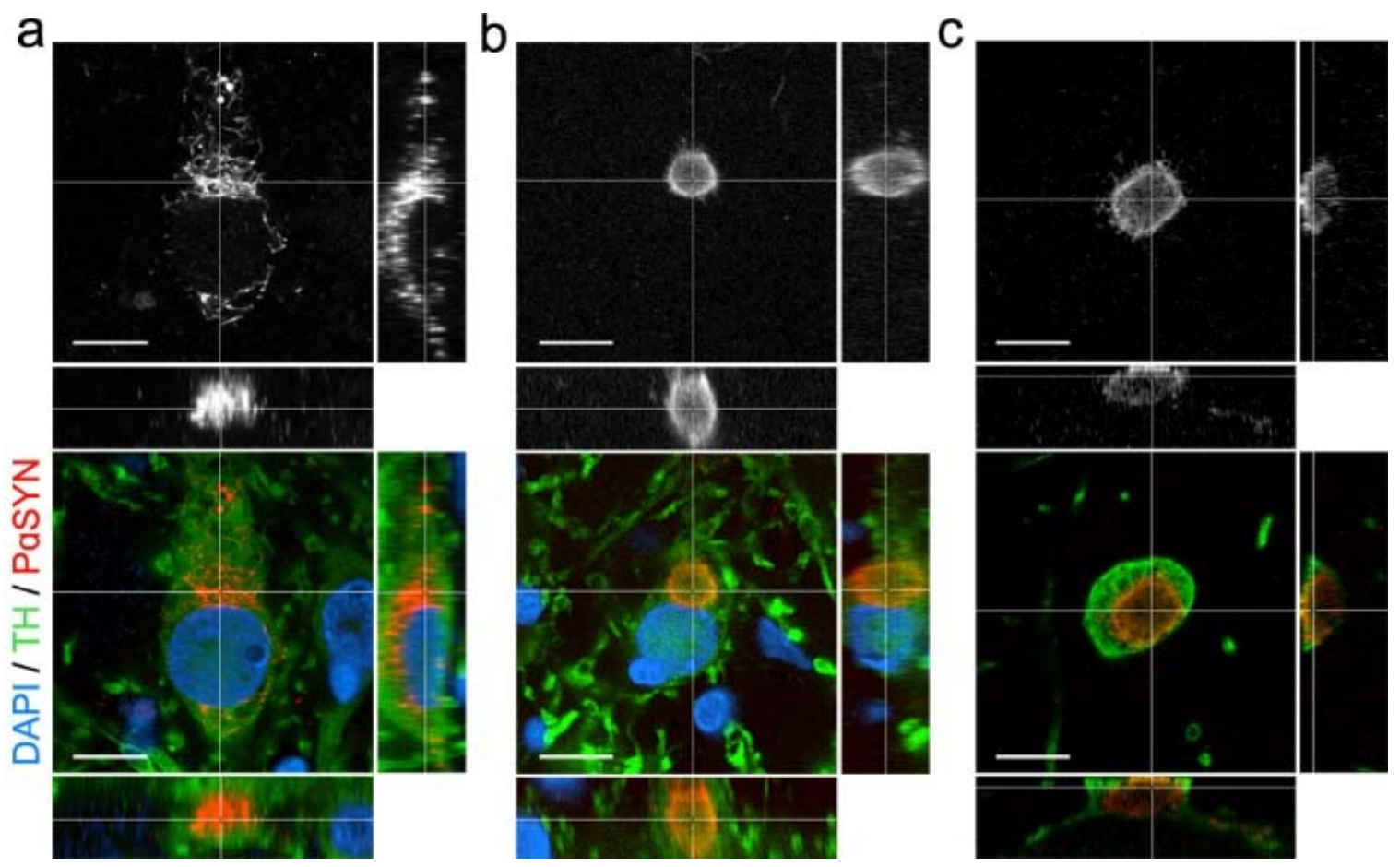

Extended Data Figure 3: Intracytoplasmic granular and nulcear inclusions
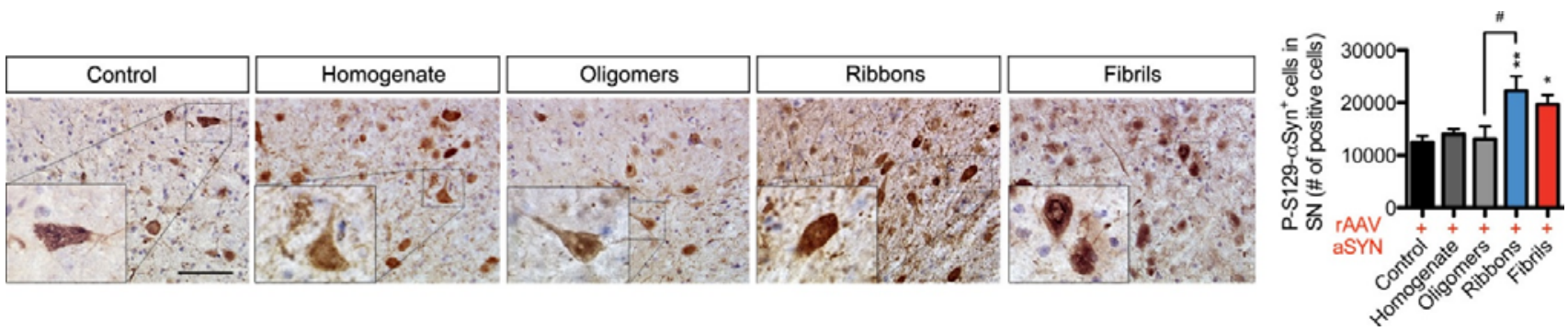

Extended Data Figure 4: Phosphorylation pattern of different alpha-SYN assemblies after combined rAAV-alpha-SYN overexpression
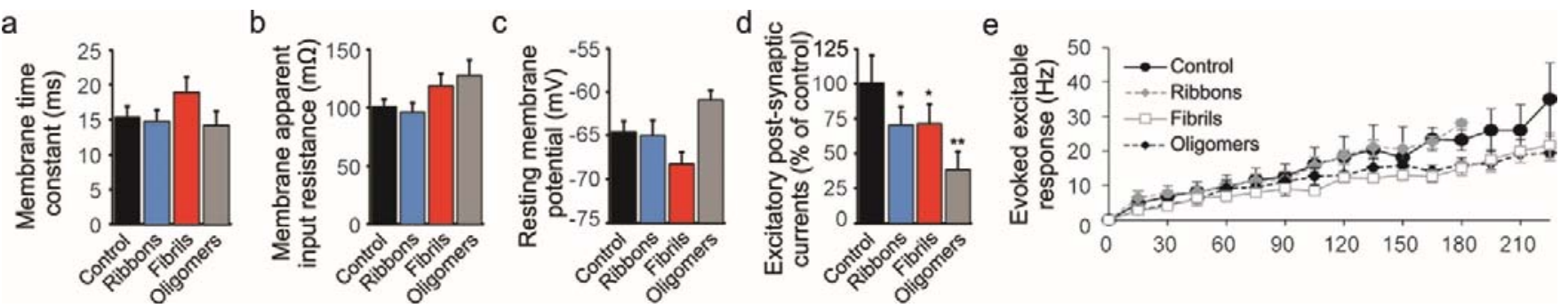

Extended Data Figure 5: Intracellular and extracellular electrophysiological recordings of early effects of alpha-SYN aggregates on single cells and on networks of neurons 


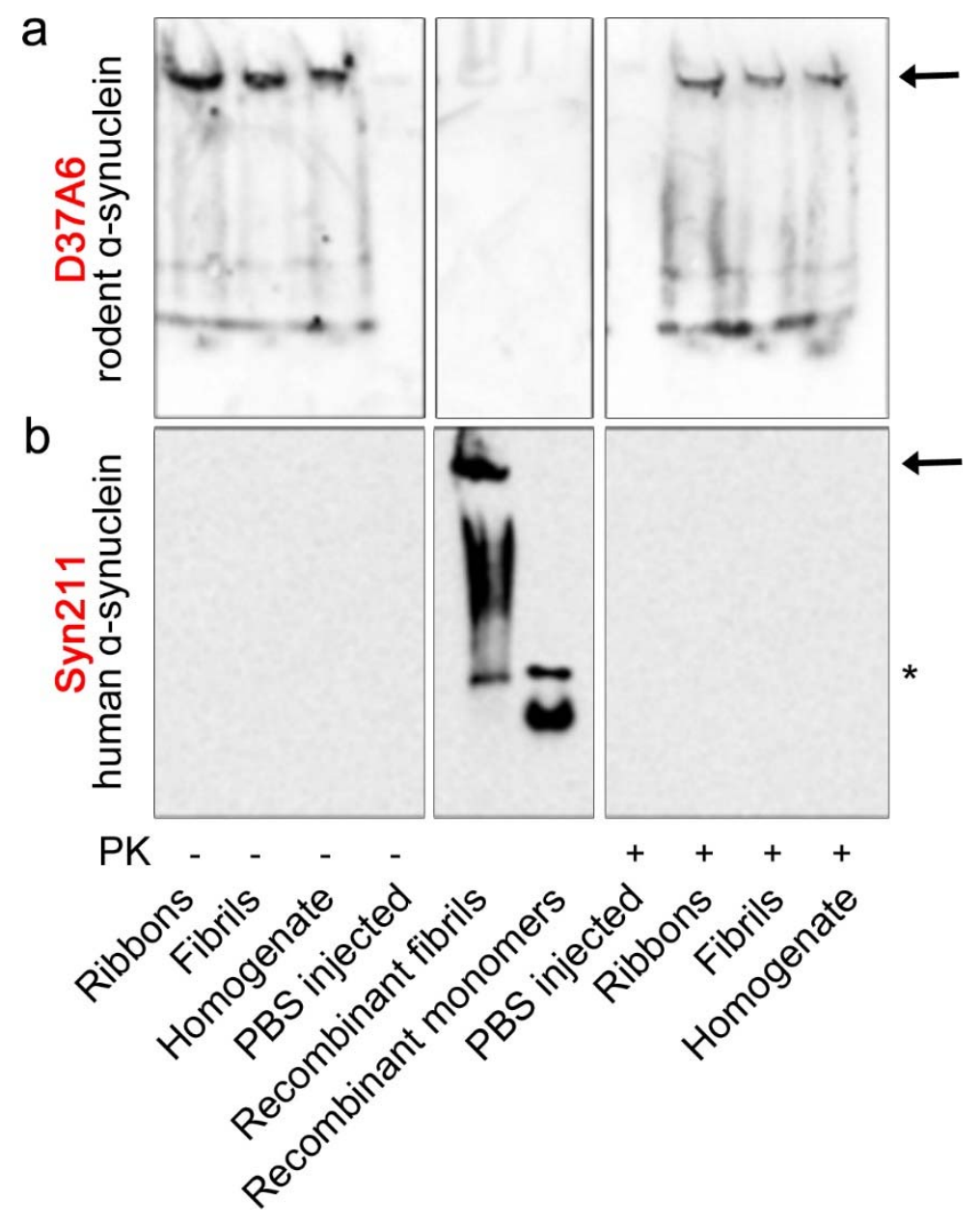

Extended Data Figure 6: Recruitment of rat endogenous alpha-SYN 
a Oligomers Fibrils Ribbons

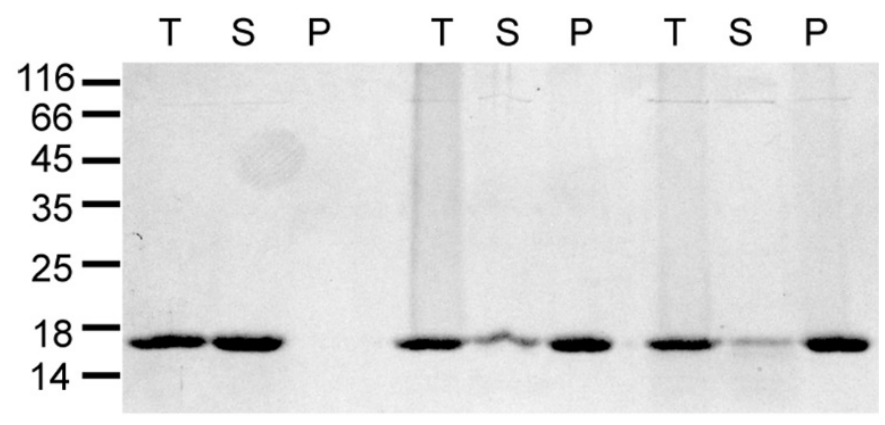

b
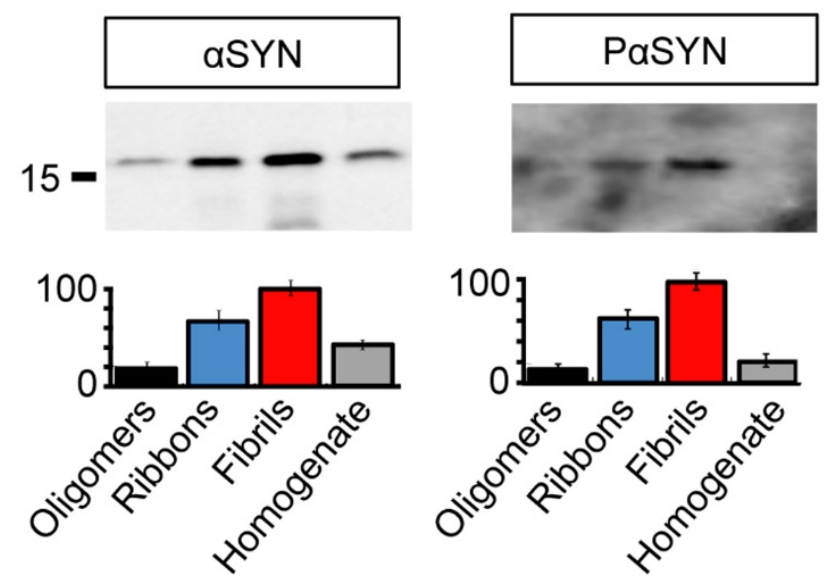

Extended Data Figure 7: Alpha-SYN strains propagate after intracerebral inoculation

a
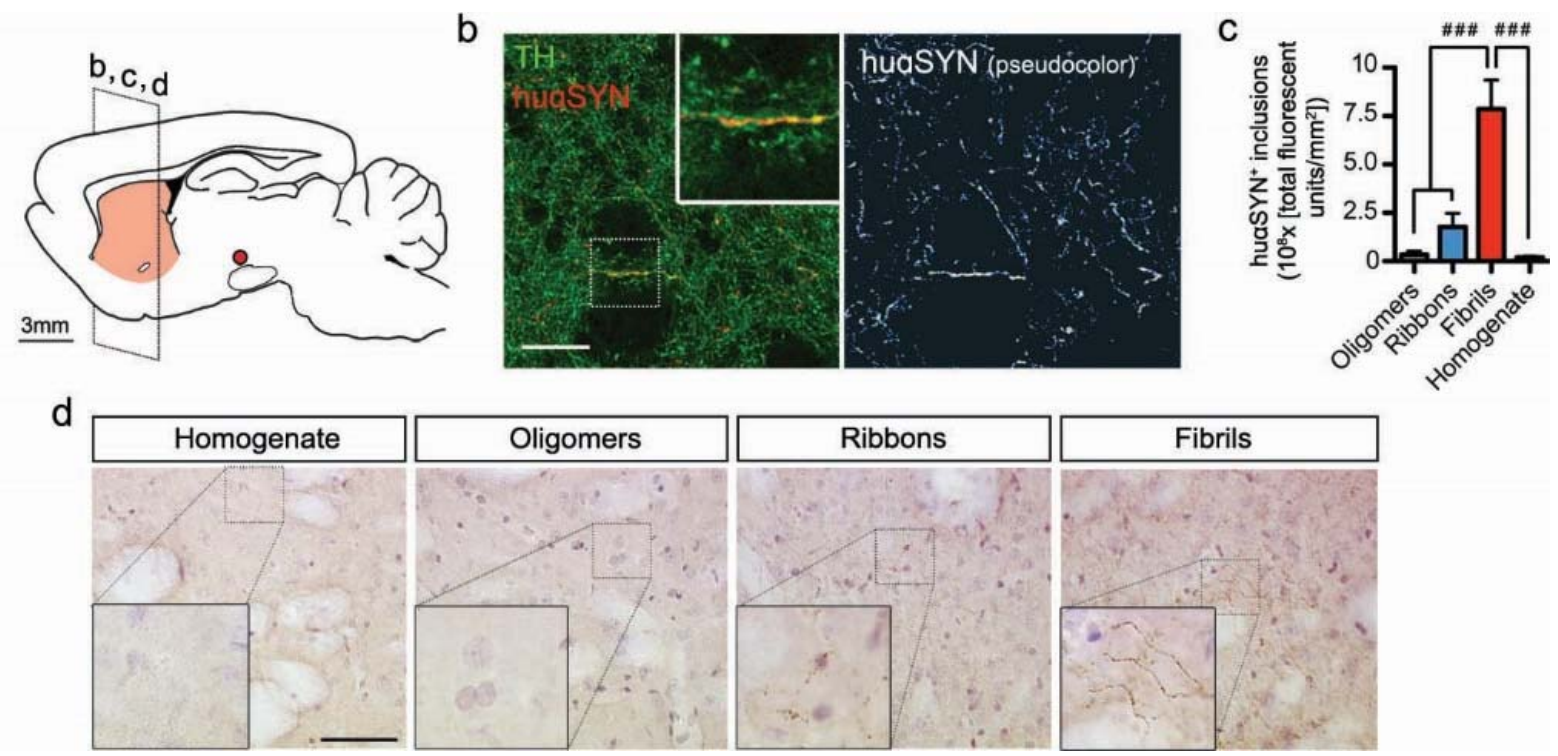

Extended Data Figure 8: Alpha-SYN fibrils persist months after intracerebral inoculation 


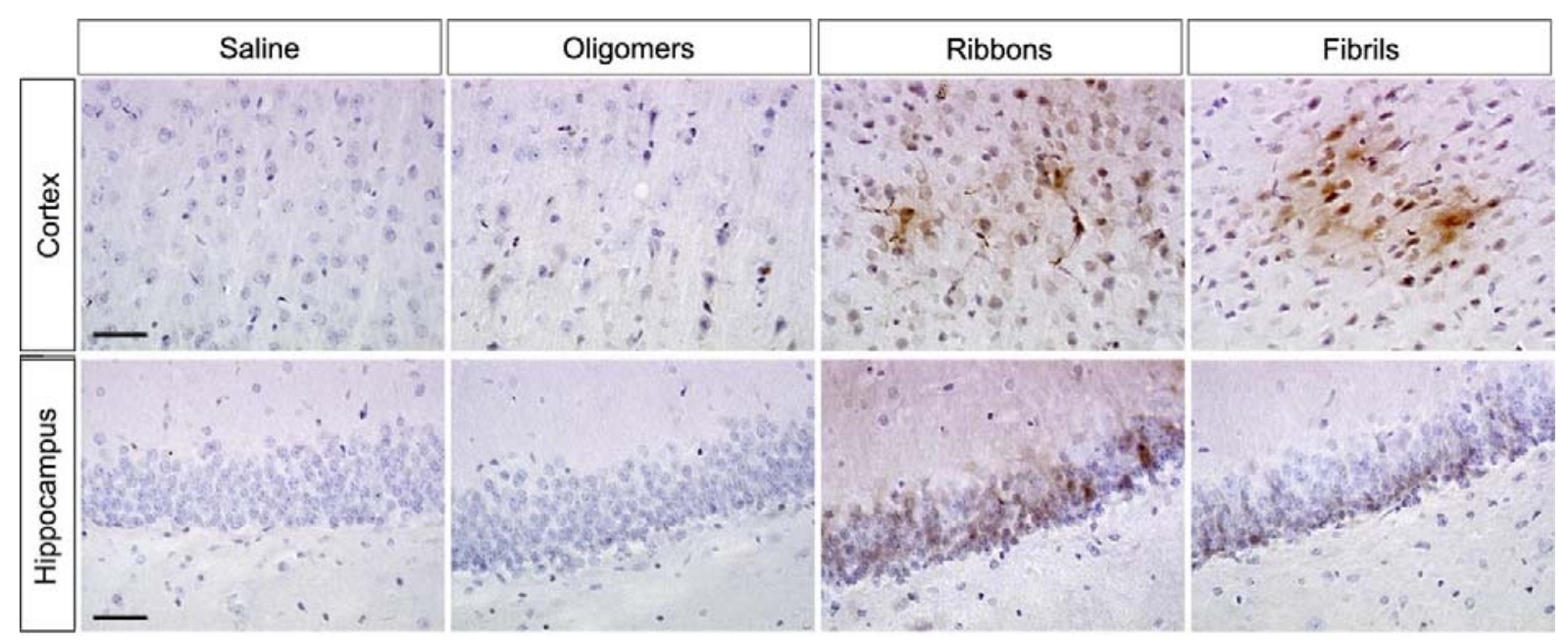

Extended Data Figure 9: Alpha-SYN ribbons and fibrils spread across the CNS after intravenous administration 

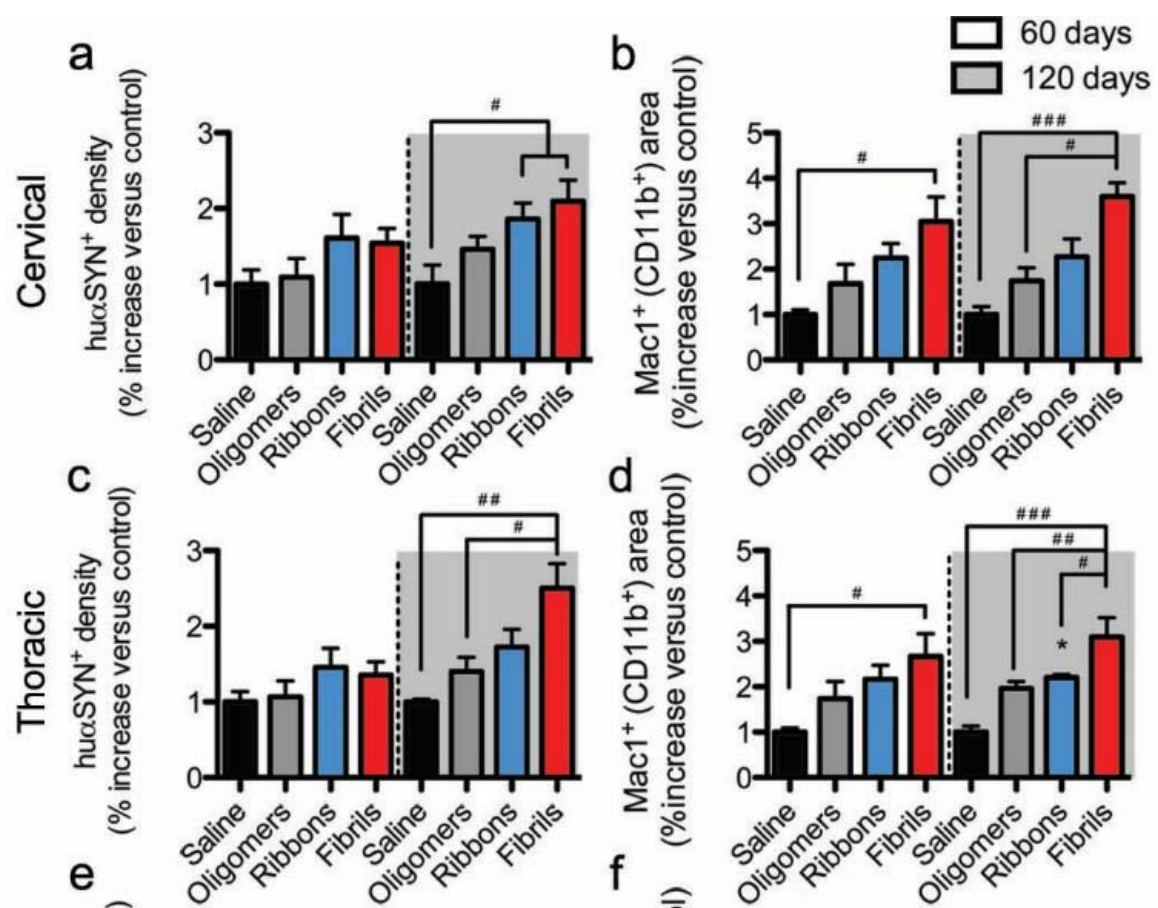

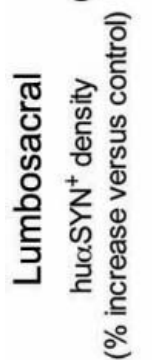

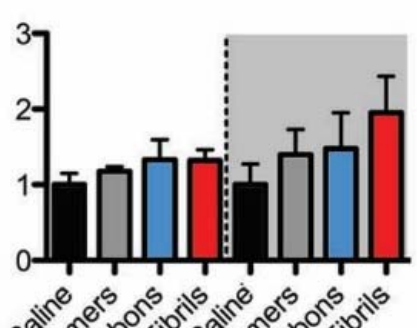

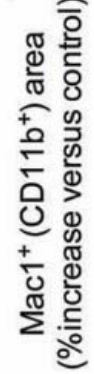
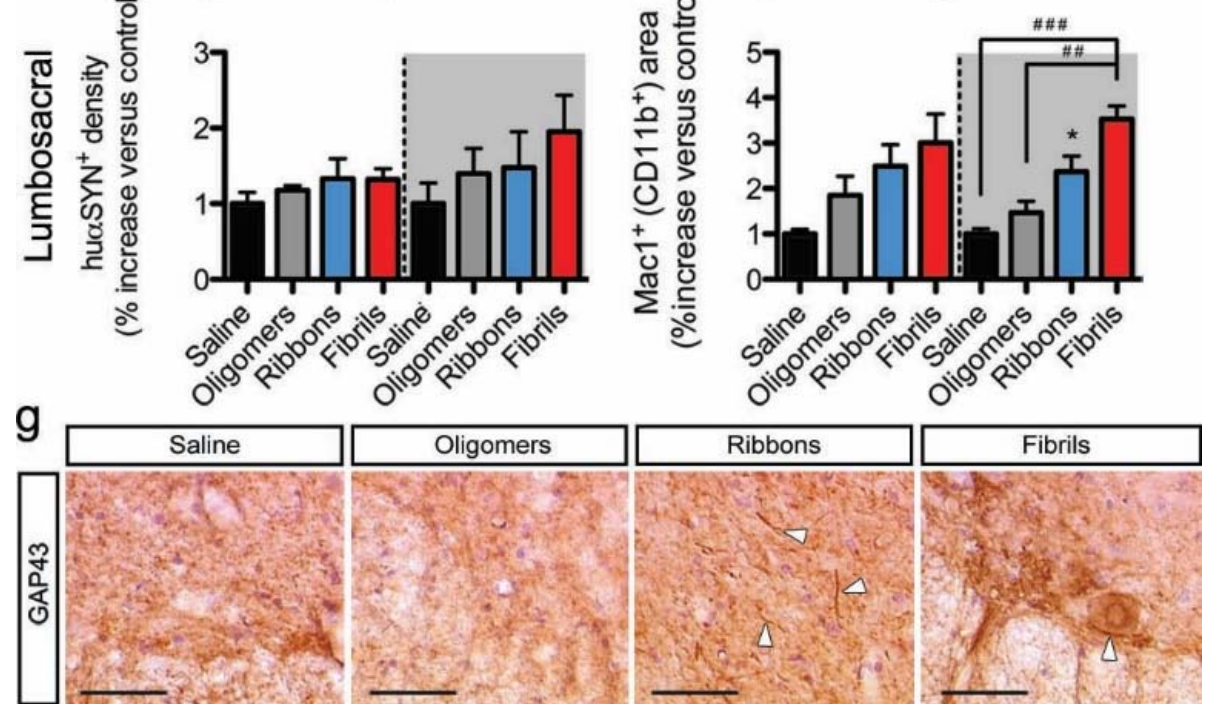

Extended Data Figure 10: Intravenous injection of alpha-SYN assemblies leads to increased microglial response throughout the spinal cord 


\section{Extended data Tables}

\begin{tabular}{|c|c|c|c|c|c|}
\hline $\begin{array}{l}\text { Injection } \\
\text { site }\end{array}$ & Coordinates & [aSYN] & $\begin{array}{l}\text { [aSYN] } \\
\text { injected }\end{array}$ & $\begin{array}{c}\text { Supplemented } \\
\text { with }\end{array}$ & Used in \\
\hline $\begin{array}{c}\text { Substantia } \\
\text { Nigra }\end{array}$ & $\begin{array}{l}\text { AP: }-5.3 \\
\text { LAT: }-2.0 \\
\text { DV: }-7.2\end{array}$ & $5 \mu \mathrm{g} / \mu \mathrm{l}(350 \mu \mathrm{M})$ & $\begin{array}{c}2 \mu \mathrm{l}(10 \mu \mathrm{g} \text { in } \\
\text { total })\end{array}$ & $1 \mu$ l saline & $\begin{array}{l}\text { Figure } 1 \\
\text { Figure 2a,b,f,h } \\
\text { Extended Data } \\
\text { Figure } 2 \\
\text { Extended Data } \\
\text { Figure } 3\end{array}$ \\
\hline $\begin{array}{l}\text { Substantia } \\
\text { Nigra }\end{array}$ & $\begin{array}{l}\text { AP: }-5.3 \\
\text { LAT: }-2.0 \\
\text { DV: }-7.2\end{array}$ & $5 \mu \mathrm{g} / \mu \mathrm{l}(350 \mu \mathrm{M})$ & $\begin{array}{c}2 \mu \mathrm{l}(10 \mu \mathrm{g} \text { in } \\
\text { total })\end{array}$ & $\begin{array}{c}1 \mu \mathrm{l} \text { of } 3.0 \times 10^{10} \\
\text { GC/ml A53T } \\
\text { aSYN rAAV2/7 }\end{array}$ & $\begin{array}{l}\text { Figure } 2 \mathrm{c}, \mathrm{d}, \mathrm{g}, \mathrm{h} \\
\text { Extended Data } \\
\text { Figure } 4\end{array}$ \\
\hline Striatum & $\begin{array}{c}\text { AP: } 0 \\
\text { LAT: }-0.28 \\
\text { DV: }-0.51,- \\
0.64,-0.77\end{array}$ & $10 \mu \mathrm{g} / \mu \mathrm{l}(700 \mu \mathrm{M})$ & $\begin{array}{c}1.33 \mu \mathrm{l} / \mathrm{injection} \\
\text { spot }(40 \mu \mathrm{g} \text { in } \\
\text { total })\end{array}$ & $\begin{array}{c}0.33 \mu \mathrm{l} \\
\text { saline/injection } \\
\text { spot }\end{array}$ & $\begin{array}{l}\text { Extended Data } \\
\text { Figure } 6 \mathrm{~b} \\
\text { Extended Data } \\
\text { Figure } 7 \\
\text { Extended Data } \\
\text { Figure } 8\end{array}$ \\
\hline Striatum & $\begin{array}{c}\text { AP: } 0 \\
\text { LAT: }-0.28 \\
\text { DV: }-0.51,- \\
0.64,-0.77\end{array}$ & $10 \mu \mathrm{g} / \mu \mathrm{l}(700 \mu \mathrm{M})$ & $\begin{array}{c}1.33 \mu \mathrm{l} / \text { injection } \\
\text { spot }(40 \mu \mathrm{g} \text { in } \\
\text { total })\end{array}$ & $\begin{array}{c}0.33 \mu \mathrm{l} \text { of } \\
3.0 \times 10^{10} \mathrm{GC} / \mathrm{ml} \\
\text { A53T aSYN } \\
\text { rAAV2/7/injection } \\
\text { spot }\end{array}$ & Figure 3 \\
\hline Tail & intravenous & $5 \mu \mathrm{g} / \mu \mathrm{l}(350 \mu \mathrm{M})$ & $\begin{array}{c}2 \mu \mathrm{l}(10 \mu \mathrm{g}) \\
\text { every } 2 \text { weeks } \\
\text { during } 4 \text { months }\end{array}$ & $500 \mu \mathrm{l}$ of saline & $\begin{array}{l}\text { Figure } 4 \\
\text { Extended Data } \\
\quad \text { Figure } 9 \\
\text { Extended Data } \\
\text { figure } 10\end{array}$ \\
\hline
\end{tabular}

Extended data Table 1. Descriptive table of different administration routes of recombinant $\alpha S Y N$ assemblies, viral vector and combinations of aSYN assemblies and viral vectors (AP, anterioposterior; LAT, mediolateral; DV, dorsoventral). 


\begin{tabular}{|c|c|c|c|c|c|c|}
\hline & Oligomers & Oligomers+ & Ribbons & Ribbons+ & Fibrils & Fibrils+ \\
\hline Spreading & +++ & I & + & I & + & I \\
\hline Seeding & - & - & + & +++ & ++ & +++ \\
\hline Propagation & - & - & + & +++ & + & +++ \\
\hline Synaptic deficit & +++ & I & +++ & I & +++ & I \\
\hline Motor deficit & - & ++ & + & +++ & + & +++ \\
\hline DA Cell death & - & ++ & - & ++ & - & +++ \\
\hline
\end{tabular}

Extended data Table 2. Overview of the effects induced by different aSYN species in absence or presence of aSYN overexpression. Red plus (+) indicates addition of rAAV2/7 A53T aSYN viral vector (- no effect, + small effect, ++ moderate effect, +++ strong effect, / not assessed). 


\begin{tabular}{|c|c|c|c|c|c|c|c|}
\hline Antibody & Reference & Catalog nr & Clone nr & Host & $\begin{array}{l}\text { Dilution } \\
\text { (IHC) }\end{array}$ & $\begin{array}{l}\text { Dilution } \\
\text { (IF) }\end{array}$ & $\begin{array}{l}\text { Dilution } \\
\text { (WB) }\end{array}$ \\
\hline Hu aSYN & Millipore & S5566 & Syn 211 & Mse & $1: 5,000$ & $1: 1,000$ & - \\
\hline aSYN & $\begin{array}{c}\text { BD } \\
\text { Bioscience }\end{array}$ & 610787 & 42 & Mse & - & - & $1 / 2,500$ \\
\hline $\begin{array}{l}\text { Rodent } \\
\text { aSYN }\end{array}$ & $\begin{array}{c}\text { Cell } \\
\text { Signaling }\end{array}$ & 4639 & D37A6 & $\mathrm{Rb}$ & - & - & $1 / 2,500$ \\
\hline Hu aSYN & $\begin{array}{l}\text { Enzo Life } \\
\text { Sciences }\end{array}$ & $\begin{array}{l}\text { ALX-804-258- } \\
\text { L001 }\end{array}$ & $15 \mathrm{G} 7$ & Rat & $1: 100$ & $1: 50$ & - \\
\hline $\begin{array}{l}\text { P-S129 } \\
\text { aSYN }\end{array}$ & 29 & - & $11 \mathrm{~A} 5$ & Mse & $1: 1,000$ & - & $1 / 2,000$ \\
\hline BIII-tubulin & Abcam & AB52901 & EP13314 & $\mathrm{Rb}$ & - & $1: 1,000$ & - \\
\hline DARPP-32 & Santa-Cruz & SC-8483 & $\mathrm{N}-19$ & Gt & - & $1: 1,000$ & - \\
\hline $\mathrm{TH}$ & Chemicon & AB152 & - & $\mathrm{Rb}$ & $1: 10,000$ & - & - \\
\hline $\mathrm{TH}$ & $\begin{array}{l}\text { AVES labs, } \\
\text { Inc. }\end{array}$ & TYH & TH1205 & Cck & - & $1: 1,000$ & - \\
\hline Olig2 & Millipore & AB9610 & 2397064 & $\mathrm{Rb}$ & - & $1: 500$ & - \\
\hline CD11b & ABD Serotec & MCA275GA & 0212 & Rat & $1: 750$ & & - \\
\hline $\begin{array}{c}\text { P62/SQSTM } \\
1\end{array}$ & PTGLab & 55274-1-AP & - & $\mathrm{Rb}$ & - & $1: 500$ & - \\
\hline $\begin{array}{l}\text { anti-mouse- } \\
\text { biotin }\end{array}$ & $\begin{array}{c}\text { DakoCytoma } \\
\text { tion }\end{array}$ & E0433 & - & Gt & $1: 2,000$ & - & - \\
\hline $\begin{array}{l}\text { anti-rabbit- } \\
\text { biotin }\end{array}$ & $\begin{array}{c}\text { DakoCytoma } \\
\text { tion }\end{array}$ & E0432 & - & $\mathrm{Gt}$ & $1: 1,000$ & - & - \\
\hline $\begin{array}{l}\text { Alexa } 405 \\
\text { anti-mouse }\end{array}$ & $\begin{array}{l}\text { Molecular } \\
\text { Probes }\end{array}$ & A31553 & - & Gt & - & $1: 500$ & - \\
\hline $\begin{array}{l}\text { FITC anti- } \\
\text { chicken }\end{array}$ & Lucron & $703-095-155$ & - & $\mathrm{Gt}$ & - & $1: 500$ & - \\
\hline $\begin{array}{c}\text { Alexa } 488 \\
\text { anti-goat }\end{array}$ & $\begin{array}{l}\text { Molecular } \\
\text { Probes }\end{array}$ & A11055 & - & Dy & - & $1: 500$ & - \\
\hline
\end{tabular}




\begin{tabular}{|c|c|c|c|c|c|c|c|}
\hline $\begin{array}{l}\text { Alexa } 647 \\
\text { anti-mouse }\end{array}$ & $\begin{array}{l}\text { Molecular } \\
\text { Probes }\end{array}$ & A31571 & - & Dy & - & $1: 500$ & - \\
\hline Rabbit HRP & Bethyl & A120-101P & - & $\mathrm{Gt}$ & - & - & $1 / 2,500$ \\
\hline $\begin{array}{c}\text { Mouse lgG } \\
\text { HRP }\end{array}$ & GeneTex & $\begin{array}{c}\text { GTX213111- } \\
01\end{array}$ & - & $\mathrm{Gt}$ & - & - & $1 / 2,500$ \\
\hline
\end{tabular}

Extended data Table 3. Descriptive table of antibodies used throughout this study. 